Subscriber access provided by Caltech Library

\title{
Article
}

\section{Diboron- and Diaza-Doped Anthracenes and Phenanthrenes: Their Electronic Structures for Being Singlet Fission Chromophores}

\author{
Ekadashi Pradhan, Seunghoon Lee, Cheol Ho Choi, and Tao Zeng
}

J. Phys. Chem. A, Just Accepted Manuscript • DOI: 10.1021/acs.jpca.0c06915 • Publication Date (Web): 09 Sep 2020

Downloaded from pubs.acs.org on September 10, 2020

\section{Just Accepted}

"Just Accepted" manuscripts have been peer-reviewed and accepted for publication. They are posted online prior to technical editing, formatting for publication and author proofing. The American Chemical Society provides "Just Accepted" as a service to the research community to expedite the dissemination of scientific material as soon as possible after acceptance. "Just Accepted" manuscripts appear in full in PDF format accompanied by an HTML abstract. "Just Accepted" manuscripts have been fully peer reviewed, but should not be considered the official version of record. They are citable by the Digital Object Identifier (DOI@). "Just Accepted" is an optional service offered to authors. Therefore, the "Just Accepted" Web site may not include all articles that will be published in the journal. After a manuscript is technically edited and formatted, it will be removed from the "Just Accepted" Web site and published as an ASAP article. Note that technical editing may introduce minor changes to the manuscript text and/or graphics which could affect content, and all legal disclaimers and ethical guidelines that apply to the journal pertain. ACS cannot be held responsible for errors or consequences arising from the use of information contained in these "Just Accepted" manuscripts. 


\title{
Diboron- and Diaza-doped Anthracenes and Phenanthrenes: Their Electronic Structures for Being Singlet Fission Chromophores
}

\author{
Ekadashi Pradhan, ${ }^{\dagger}$ Seunghoon Lee, ${ }^{\ddagger}$ Cheol Ho Choi, ${ }^{\top}$ and Tao Zeng*,† \\ †Department of Chemistry, York University, Toronto, Ontario, M3J1P3, Canada \\ $\ddagger$ Division of Chemistry and Chemical Engineering, California Institute of Technology, \\ Pasadena, California 91125, USA \\ 【Department of Chemistry, Kyungpook National University, Daegu 702-701, South Korea \\ E-mail: tzeng@yorku.ca \\ Phone: +1 (416) 7362100 ext. 33673
}




\begin{abstract}
We used quantum chemistry methods at the levels of mixed-reference spin-flipping time-dependent density functional theory and multireference perturbation theory to study diboron- and diaza-doped anthracenes and phenanthrenes. This class of structures recently surged as potential singlet fission chromophores. We studied electronic structures of their excited states and clarify the reasons why they satisfy or fail to satisfy the energy criteria for singlet fission chromophores. Many studied structures have their $S_{1}$ states not dominated by $\mathrm{HOMO} \rightarrow \mathrm{LUMO}$ excitation, so that they cannot be described using the conventional two sites model. This is attributed to frontier orbital energy shifts induced by the doping and different charge transfer energies in different one-electron singlet excitations, or in other words, different polarizations of hole and/or particle orbitals in their $S_{1}$ and $T_{1}$ states. There is a mirror relation between the orbital energy shifts induced by diboron- and diaza-dopings, which, together with alternant hydrocarbon pairings of occupied and unoccupied orbitals, leads to more mirror relations between the excited states of the two types of doped structures.
\end{abstract}

\title{
Introduction
}

Singlet fission (SF) is the process that one molecule at singlet excited state shares its energy with a neighboring molecule at ground state and result in two molecules in triplet excited states. ${ }^{1-3}$ The number of excited states is doubled and the triplet excited states have longer lifetime. These two effects make SF a highly interesting process in photovoltaics. The process has the potential to enhance the photoelectric conversion efficiency to exceed the $\sim 30 \%$ Shockley-Queisser limit $^{4}$ and reach $\sim 50 \%$ for a single junction photovoltaic device. ${ }^{5}$ One obstacle for the advancement of exploiting SF in photovoltaics is the small pool of chromophores that can undergo this process. ${ }^{6-9}$ Actually, SF research has been "overwhelmingly limited" to the two longer polyacenes and their derivatives. ${ }^{10}$ This fact motivates the thriving studies in proposing other SF chromophores. 
In order to undergo SF, a chromophore molecule needs to satisfy the following criteria for its excited states' energies: ${ }^{1}$

$$
\begin{aligned}
& E\left(S_{1}\right)>2 E\left(T_{1}\right) \\
& E\left(T_{2}\right)>2 E\left(T_{1}\right) \\
& E\left(Q_{1}\right)>2 E\left(T_{1}\right) .
\end{aligned}
$$

" $S_{i}$ ", " $T_{i}$ " and " $Q_{i}$ " stand for $i$-th excited states within the singlet, triplet, and quintet spin manifolds, respectively. Note that all energies in the three inequalities above are excitation energies of one chromophore molecule. The inequality in Eq. 1 ensures an exoergic fission of a singlet exciton to two triplet excitons. The other two inequalities ensure endoergic and thus unfavorable recombination of two triplet excitons on two molecules to one triplet exciton or one quintet exciton on one molecule. The recombination may occur between two independent triplet excitons, whose randomly oriented spins can add up to an overall singlet, triplet, or quintet spin. ${ }^{11}$ The recombination is thus an internal conversion and does not involve spin flipping. ${ }^{1}$ Since the $Q_{1}$ state is usually higher in energy than $S_{1}$ and $T_{2}$, we can safely ignore Eq. 3 and focus on the other two criteria. If $E\left(T_{2}\right)$ is close to or even smaller than $2 E\left(T_{1}\right)$, then the two SF-generated $T_{1}$ excitons may recombine to form one $T_{2}$ exciton on one molecule and the other partner in $S_{0}$ state. However, $T_{2}$ often involves $\mathrm{HOMO} \rightarrow \mathrm{LUMO}+1$ or $\mathrm{HOMO}-1 \rightarrow$ LUMO excitation, while $T_{1}$ involves $\mathrm{HOMO} \rightarrow \mathrm{LUMO}$ excitation. The difference in orbital energy gaps is usually large enough to guarantee the inequality in Eq. 2. Therefore, Eq. 2 is often considered as a secondary criterion compared to Eq. 1. However, this is not always the case, when there is orbital pseudo-degeneracy between HOMO-1 and HOMO, or between LUMO and LUMO+1. This situation does occur for some molecules considered in this work.

Eq. 1 is the primary criterion for SF chromophores and has been the focus of chromophores designing. Considering thermal activation, this criterion can be relaxed and 
molecules with $E\left(S_{1}\right)$ slightly smaller than $2 E\left(T_{1}\right)$ may also undergo SF. ${ }^{1}$ Paci et al. first pointed out that diradicaloids and large polycyclic aromatic hydrocarbons (PAHs) are likely to satisfy the criterion. ${ }^{12}$ Later, Nakano and coworkers used a symmetric two sites model to associate the $E\left(S_{1}\right) \sim 2 E\left(T_{1}\right)$ relation solely to diradical character; ${ }^{13-16}$ PAHs satisfy Eq. 1 when they are large enough to have adequate diradical character. This association explains the rarity of SF chromophores. While too little diradical character makes $E\left(S_{1}\right)<2 E\left(T_{1}\right)$, too much diradical character makes a molecule highly reactive and unstable. We hence need to finely tune diradical character of a molecule to make it a stable SF chromophore, which is not easier than "finding a few needles in a haystack." ${ }^{17}$ Difficult yet possible. The beacon of diradical character has guided the community of SF to design a few chromophores. ${ }^{7,9,17}$ 1,3-Diphenylisoenzofuran is a typical example and it has been synthesized and exhibits SF behavior. ${ }^{18-21}$ For PAHs, diradical character is closely related to ground state antiaromaticity, and so is the $E\left(S_{1}\right) \sim 2 E\left(T_{1}\right)$ relation. ${ }^{14}$ Recently, more designs of SF chromophores based on ground state antiaromaticity have been proposed. ${ }^{22-24}$ Using the two sites model, the Nakano group also pointed out that the exchange integral $K_{a, b}$ is an extra knob for tuning the $E\left(S_{1}\right) \sim 2 E\left(T_{1}\right)$ relation, where $a$ and $b$ stand for the localized orbitals that are transformed from HOMO and LUMO as $\sqrt{\frac{1}{2}}$ (HOMO \pm LUMO). ${ }^{25,26}$

The essences of the two sites model are summarized as follows. (1) The HOMO and LUMO are of different irreducible representations (IRREPs) of the chromophore's point group, e.g., of " $g$ " and " $u$ " $I$-parities and/or " $A$ " and " $B$ " $C_{2}$-parities. They are viewed as linear combinations of two "localized" "nonbonding" asymmetric orbitals $a$ and $b$ (vide supra). These two sites are symmetry images of each other and hence have the same energy. The quotation marks emphasize that $a$ and $b$ might themselves be delocalized over a few atoms, and there may be bonding and/or antibonding characters within $a$ and $b$. However, it is the bonding and antibonding interactions between $a$ and $b$ that open the HOMO-LUMO gap $\left(\Delta_{H, L}\right)$. (2) With the symmetry, the 2 electrons and 2 orbitals configuration interaction (2o2e CI) problem can be solved exactly. There are four configuration states: the singlet 

symmetric and antisymmetric charge-transfer states (SC and $\mathrm{AC}$ ) with two electrons solely on $a$ or on $b . S_{0}\left(S_{2}\right)$ is the bonding (antibonding) mixture of SD and SC, $S_{1}$ is of pure AC character, and $T_{1}$ is of pure $\mathrm{T}$ character. (3) $2 K_{H, L}=\left(J_{a, a}-J_{a, b}\right)$, where $J$ denotes Coulomb integral of the orbitals in subscript. $2 K_{H, L}$ is thus the electrostatic energy cost of charge transfer $(\mathrm{CT})$ from the configuration with one electron on each site to that with both electrons on one site. The energy difference between $\mathrm{T}$ and $\mathrm{AC}$ (i.e, the $E\left(T_{1}\right)-E\left(S_{1}\right)$ gap) is $2 K_{H, L}$. A large $K_{H, L}$ suggests a higher $E\left(S_{1}\right)$ and a lower $E\left(T_{1}\right)$, and hence a better propensity to satisfy Eq. 1. (4) $2 K_{H, L}$ is also the energy gap between SD and SC, whose interaction matrix element is $\frac{1}{2} \Delta_{H, L}$. Therefore, a small $\Delta_{H, L} / K_{H, L}$ ratio indicates a smaller interaction between $\mathrm{SD}$ and $\mathrm{SC}$, and thus a higher $E\left(S_{0}\right)$. Relatively, this implies lower $E\left(T_{1}\right)$ and $E\left(S_{1}\right)$ together. Since the reduction of $E\left(T_{1}\right)$ counts twice in the Eq. 1 inequality, the together lowerings of $E\left(T_{1}\right)$ and $E\left(S_{1}\right)$ imply a better propensity to satisfy the inequality. Considering all these effects of $K_{H, L}$ and $\Delta_{H, L}$, their ratio $K_{H, L} / \Delta_{H, L}$ (which implies diradical character, and is also related to the MP2 perturbation amplitude for the $\mathrm{HOMO} \rightrightarrows \mathrm{LUMO}$ excitation) is a determining factor for satisfying Eq. 1. (5) $\mathrm{T}$ and $\mathrm{AC}\left(T_{1}\right.$ and $S_{1}$ ) are $2 K_{a, b}$ lower in energy than $\mathrm{SD}$ and $\mathrm{SC}$, respectively. Therefore, a larger $K_{a, b}$ suggests lower $E\left(S_{1}\right)$ and $E\left(T_{1}\right)$ together. Again, the $E\left(T_{1}\right)$ lowering counts twice in Eq. 1, and $K_{a, b}$ is also a factor that determines the propensity to satisfy Eq. 1.

The mathematical expressions of the ground and excited states' energies taking $\Delta_{H, L}$, $K_{H, L}$, and $K_{a, b}$ as variables are shown in Refs. $13-16,25,27,28$. Since the impacts of the three parameters on the energies are conceptually transferrable to calculations more accurate than the 2o2e CI treatment, a qualitative description of the impacts given in the previous paragraph is more helpful, we believe, for understanding why a molecule satisfies or does not satisfy Eq. 1. However, if the two sites model becomes invalid, i.e., when the low-lying excited states involve excitations beyond the 2o2e small space, diradical character and $K_{a, b}$ are not the only determining factors for their energies. On the other hand, as long as the low-lying 
excited states can be described using excitations within the 2o2e space, diradical character and $K_{a, b}$ are the main determining factors. For those molecules, we may also use ground state antiaromaticity to explain their $E\left(S_{1}\right) \sim 2 E\left(T_{1}\right)$ relation. But this explanation must be consistent with the one based on diradical character and $K_{a, b}$. A few of the diboron- (2B-) and diaza-doped (2N-doped) molecules that we study in this work cannot be described using the two sites model. Although the 2B-doped molecules have been recently investigated as potential SF chromophores, ${ }^{29}$ we see a necessity to look back at those molecules, and explain their unexpected properties of excited states.

The motivations of doping two B atoms into anthracene and phenanthrene are as follows. (1) Anthracene is historically related to SF. The first indirect observation of SF is through the delayed fluorescence of anthracene crystal. ${ }^{30}$ (2) Unlike its longer analogues tetracene and pentacene, ${ }^{31-35}$ anthracene and its isomer phenanthrene are small PAHs that do not satisfy Eq. 1. The indirectly observed SF of anthracene crystal is not thermodynamically favorable and cannot compete with other excited state dynamics channels. Chemical tailoring on the pristine molecules can be employed to design SF chromophores. (3) The 2B-dopings convert the two $4 n+2$ systems to $4 n$ systems. The associated antiaromaticity implies high diradical character, low-lying $T_{1}$ state, and consequently the satisfying of Eq. 1. This is especially true if the $\mathrm{C}$ moiety(ies) of a doped molecule possesses (possess) a clear diradical structure. ${ }^{26,36,37}$ (4) Anthracene and phenanthrene are representatives of polyacenes and phenacenes, respectively. It is of interest to investigate their different responses to the dopings. (5) $2 \mathrm{~N}$-dopings also convert $4 n+2$ systems to $4 n$ systems, and may also lead to SF chromophores. Also, it is of interest to study the differences and similarities between doping donors $(\mathrm{N})$ and acceptors $(\mathrm{B})$.

The present study is inspired by the pioneering work of Stoycheva et al. ${ }^{29}$ on 2 B-doped anthracenes and phenanthrenes. The 2B- and 2N-doped phenanthrenes were also included in a more recent study by the Nakano group, ${ }^{26}$ Most of our calculated results are consistent with those reported in Refs. 26 and 29. Still, the present study provides another perspective 


\section{Computational methods}

Since structural reorganization after excitation occurs at a similar or even faster time scale than $\mathrm{SF}$, we optimized structures for $S_{0}, S_{1}, T_{1}$, and $T_{2}$ states of all studied molecules, and used the minimum-to-minimum (i.e., adiabatic) $E\left(S_{1}\right) \mathrm{s}, E\left(T_{1}\right) \mathrm{s}$, and $E\left(T_{2}\right) \mathrm{s}$ to judge whether the molecules satisfy the two inequalities. Structural optimizations and subsequent hessian calculations were performed using the mixed-reference spin-flipping time-dependent density functional theory (MRSF-TDDFT ${ }^{38,39}$ ) method, with the BHHLYP ${ }^{40,41}$ functional. All the optimized structures have no imaginary frequency. At the optimized structures, nelectron valence state perturbation theory ${ }^{42-44}$ (NEVPT2) calculations were performed to determine the excitation energies. Four singlet and three triplet states are averaged equally in the NEVPT2 calculations. Convergence of $E\left(S_{1}\right), E\left(S_{2}\right), E\left(T_{1}\right)$, and $E\left(T_{2}\right)$ with respect to the number of states being averaged was reached, except for the AB3B7 molecule, which requires one more singlet state in the average. Both nondynamic and dynamic correlations between electrons are treated by the MRSF-TDDFT and NEVPT2 methods. The NEVPT2 single point energies were corrected by the zero point energies (ZPEs) calculated at the MRSF-TDDFT level. Without further specification, all reported excitation energies are the ZPE-corrected 0-0 energies. All calculations were performed using the cc-pVDZ basis set. ${ }^{45}$ Test calculations using the cc-pVTZ basis set resulted in $\leq 0.02 \mathrm{eV}$ differences in excitation energies (Section S1 in the Supporting Information). Convergence with respect to basis set was reached. We also performed general multi-configuration quasi-degenerate perturbation theory ${ }^{46-48}$ (GMC-QDPT) calculations to obtain the wave functions of the ground and excited states, and analyzed them to understand how the dopings modify the 
electronic structures of the molecules.

A 12 valence $\pi$ orbitals 12 valence electrons (12o12e) complete active space is used for the NEVPT2 calculations. For the 2B-doped molecules, the two leftover valence $\pi$ orbitals feature the $\mathrm{B}$ empty $p_{z}$ orbitals with the most substantial antibonding interaction with surrounding $\mathrm{C}$ atoms and are highest in energy among the $\pi$ valence. For the $2 \mathrm{~N}$-doped molecules, the leftover $\pi$ orbitals feature the $\mathrm{N}$ lone pair character with the most substantial bonding character with surrounding $\mathrm{C}$ atoms and are lowest in energy. It is reasonable to exclude them from the active space, when it is too costly to include them, and leave them to the perturbation treatment of dynamic correlations. Actually, the same 12o12e active space was successfully employed by Kawashima et al to calculate excitation energies of anthracene. ${ }^{49}$ GAMESS-US ${ }^{50,51}$ was used for the MRSF-TDDFT and GMC-QDPT calculations. ORCA ${ }^{52}$ was used for the NEVPT2 calculations. The GMC-QDPT wave functions are constructed using state-averaged canonical orbitals, which are thus used for wave function analysis. The energies of the active orbitals that correspond to unoccupied orbitals of ground state are known to be much higher than those obtained from DFT calculations. However, the consequent larger energy gaps between occupied and unoccupied orbitals do not impair our qualitative, yet indicative analysis, which is focused on the overall trends of orbital energies' changes. Natural orbitals obtained for ground states of the molecules are used to discuss their diradical characters and assign Lewis structures. Most of the orbital plottings are given in the Supporting Information, where the figures are labeled as Figure S1, Figure S2, etc.

The $\mathrm{C}$ positions of the anthracene and phenanthrene are labeled with the IUPAC numberings. The numberings are shown in Figure 1. Following the convention in Ref. 29, the doped molecules are labeled as $\mathbf{A B} n \mathbf{B} m, \mathbf{A N} n \mathbf{N} m, \mathbf{P B} n \mathbf{B} m$, and $\mathbf{P N} n \mathbf{N} m$. "A" and "P" indicate the prestine anthracene and phenanthrene. $n$ and $m$ indicate the doping positions. Throughout this paper, $\rightarrow$ is used to represent single-electron excitations and $\rightrightarrows$ doubleelectron excitations. Suppose that the $\mathrm{HOMO} \rightarrow \mathrm{LUMO}$ configuration contributes $p_{H L} \%$ to $S_{1}$ and another configuration that contributes most significantly to $S_{1}$ has the percentage of 
A
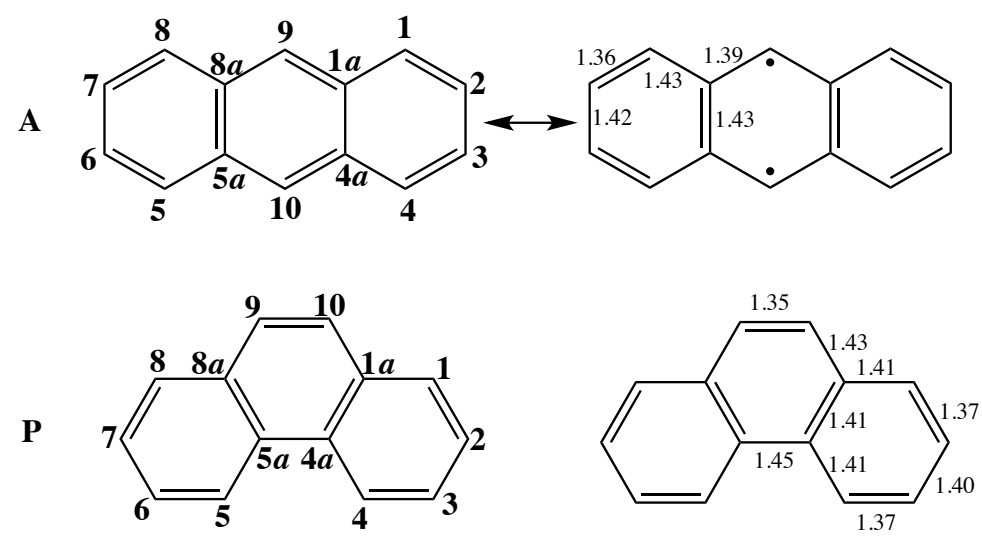

Figure 1: Anthracene (A) and phenanthrene (P), their atom numberings, and bond lengths (in $\AA$ ). Shown for anthracene is also a diradical Lewis structure contained in the molecule's ground state.

Within the closed-shell Hartree-Fock approximation, the energy of a singlet excitation from an occupied orbital $i$ to an unoccupied orbital $j^{\prime}$ is $\Delta_{i j^{\prime}}-J_{i, j^{\prime}}+2 K_{i, j^{\prime}}$, and the triplet excitation energy is $\Delta_{i, j^{\prime}}-J_{i, j^{\prime}} . \Delta_{i, j^{\prime}}$ indicates the energy cost for promoting an electron from an orbital with more bonding character to an orbital with more antibonding character. $J_{i, j^{\prime}}$ is the Coulombic binding energy exerted by a hole in $i$ onto an electron in $j^{\prime}$. In a state-averaged CASSCF calculation, this binding energy is partially absorbed in orbital energies and thus $\Delta_{i, j^{\prime}}$ as well. This is because the density matrix of the $i \rightarrow j^{\prime}$ configuration is also included in the state-averaging for the construction of Fock operator. Therefore, we ignore this term in the following discussion. The $2 K_{i j^{\prime}}$ reflects the different electron correlations in the singlet and triplet. In general, $2 K_{i j^{\prime}}=\frac{1}{2}\left(J_{a, a}+J_{b, b}\right)-J_{a, b}$, with $a$ and $b$ being the localized orbitals $\frac{1}{\sqrt{2}}\left(i \pm j^{\prime}\right) .2 K_{i j^{\prime}}$ hence reflects the averaged energy cost of cramming two electrons on one 
site rather than having one electron on each site. The CT description of singlet excitation holds even for two asymmetric sites. The aforementioned $2 K_{H, L}=J_{a, a}-J_{a, b}$ is a special case with the $J_{a, a}=J_{b, b}$ symmetry. Henceforth, Subscripts $a$ and $b$ in $K_{a, b}$ solely denote localized orbitals transformed from HOMO and LUMO.

\section{Results and discussions}

We ran calculations for all the 2B-doped molecules considered in Ref. 29 except AB9B10 and AB5B8. These two molecules feature typical closed-shell characters in their ground states. They are far from satisfying Eq. 1 and are not worth further studying in the context of SF. The 2N-doped analogues AN9N10 and AN5N8 are also not considered. We first present the calculated results of anthracene and phenanthrene in Supporting Information (SI, Section S2), which are references for the discussions of the doped molecules. In short, with not enough diradical characters, both molecules do not satisfy Eq. 1. Anthracene can be described using the two sites model as both its $S_{1}$ and $T_{1}$ are dominated by $\mathrm{HOMO} \rightarrow$ LUMO excitation. Contrarily, the $S_{1}$ of phenanthrene is dominated by equal mixing of $\mathrm{HOMO} \rightarrow \mathrm{LUMO}+1$ and $\mathrm{HOMO}-1 \rightarrow$ LUMO excitations; the two sites model is inapplicable. The small $\Delta_{H-1, H}$ and $\Delta_{L, L+1}$ gaps, the smaller $K_{H-1, L}$ and $K_{H, L+1}$ than the $K_{H, L}$ integral, and the configuration interaction between the $\mathrm{HOMO} \rightarrow \mathrm{LUMO}+1$ and HOMO-1 $\rightarrow$ LUMO configurations are responsible for the unconventional $S_{1}$ of phenanthrene.

\section{The 2B- and 2N-dopings on orbital energies}

The dopings significantly modify the orbital energies, which are shown in Figure $2 . \Delta_{H, L} \mathrm{~S}$ are all substantially reduced compared to those of the undoped molecules. This is as expected for the $4 n+2$ to $4 n$ conversion and the associated enhancement of diradical character. The general enhancement of diradical character is clearly shown by the $n_{\text {LuNo }}$ plottings in 
Figure 3(a). AN1 $a \mathbf{N} 8 a$ is an exception in the orbital energy and $n_{\text {LUNO }}$ diagrams and will be discussed later. The most significant changes in Figure 2 are the decrease of LUMO energies of the 2B-doped molecules and the increase of the HOMO energies of the $2 \mathrm{~N}$-doped molecules, which are mostly responsible for the $\Delta_{H, L}$ reductions. These general trends can be explained using the general knowledge of $p$ - and $n$-type semiconductors: the doping of acceptor (B here) inserts empty orbitals in band gap and the doping of donor ( $\mathrm{N}$ here) inserts occupied orbitals. Compared in Figure 3(b) are the decreases of LUMO energies of the 2Bdoped molecules and the increases of HOMO energies of the $2 \mathrm{~N}$-doped molecules. There is an interesting mirror relation: the LUMO energy decreases of the 2B-dopings have similar magnitudes as the HOMO energy increases of the $2 \mathrm{~N}$-dopings. This indicates that the $\mathrm{B}$ and $\mathrm{N}$ dopants have similar intensities in modifying electronic structure, but in opposite directions.
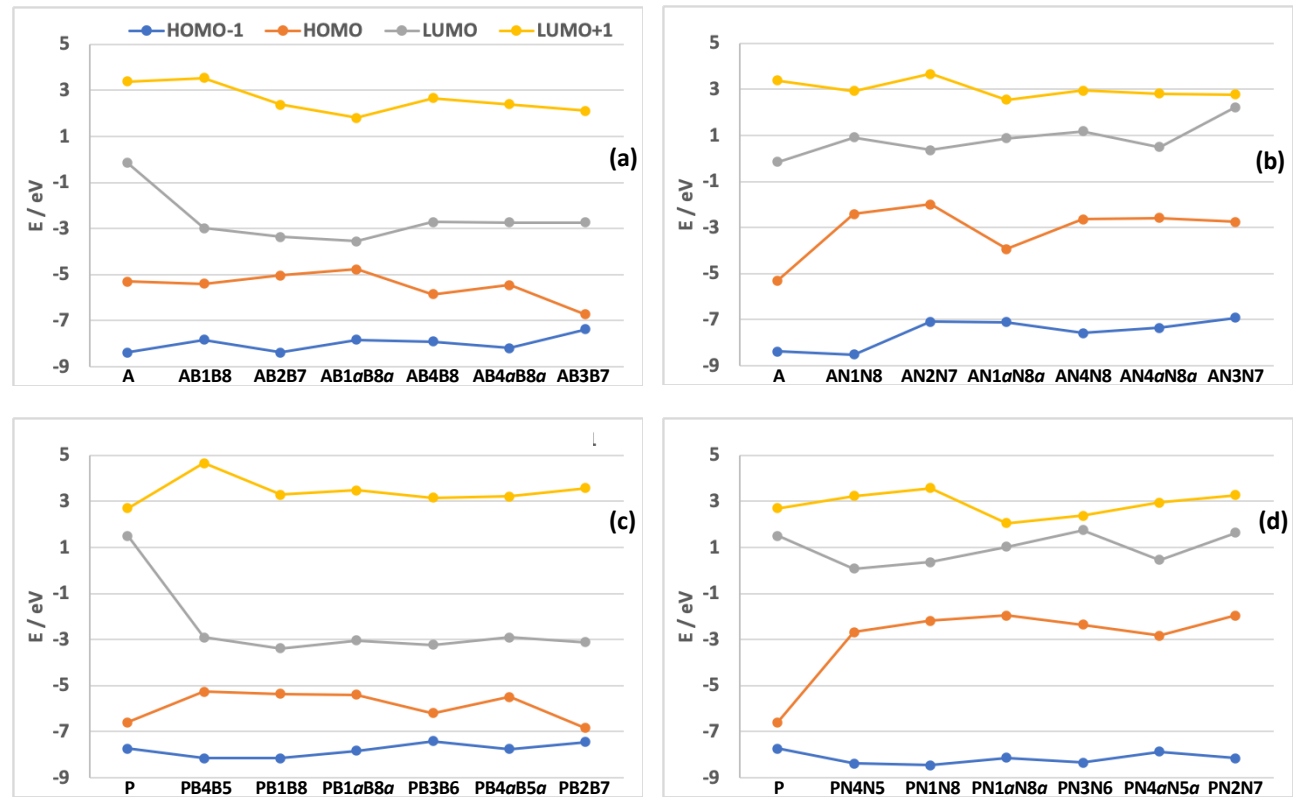

Figure 2: Canonical orbital energies of HOMO-1 to LUMO +1 of all molecules studied in this work.

There are more changes beyond the $\Delta_{H, L}$ reductions. The lower LUMO eneriges of the 2B-doped molecules also reduce their $\Delta_{H-1, L} \mathrm{~S}$. For the 2B-doped anthracenes, such a gap change may lower the energy of the HOMO-1 $\rightarrow$ LUMO configuration and, given ap- 
propriate symmetry, significantly enhance its contribution to the $S_{1}$ state. Consequently, it may invalidate the two sites model, which is applicable for anthracene. Similarly, the $2 \mathrm{~N}$-dopings reduce $\Delta_{H, L+1} \mathrm{~S}$ and may raise the percentage of the $\mathrm{HOMO} \rightarrow \mathrm{LUMO}+1$ configuration in $S_{1}$, invalidating the two sites model for the $2 \mathrm{~N}$-doped anthracenes. An opposite situation may be seen for the doped phenanthrenes. The $\Delta_{H, L}$ reduction is likely to make the $\mathrm{HOMO} \rightarrow$ LUMO configuration dominates the $S_{1}$ states of the doped phenanthrenes. Also, the $\Delta_{L, L+1} \mathrm{~S}$ are enlarged from $1.2 \mathrm{eV}$ of phenanthrenes to $>6.5 \mathrm{eV}$ in the $2 \mathrm{~B}$-doped analogues. The HOMO $\rightarrow \mathrm{LUMO}+1$ configuration must contribute less to $S_{1}$. For the $2 \mathrm{~N}-$ doped phenanthrenes, the $\Delta_{H-1, H} \mathrm{~s}$ are enlarged from $1.1 \mathrm{eV}$ to $>5.0 \mathrm{eV}$, diminishing the HOMO-1 $\rightarrow$ LUMO contribution to $S_{1}$. With these considerations, the two sites model, which is inapplicable for phenanthrene, may be more applicable for the doped phenanthrenes.

The orbital energy gap changes are not the only determining factors for excited states, especially for one-electron singlet excited states. The CT energy is another factor. Therefore, the spatial overlaps between the relevant localized orbitals also determine the percentages of those non-HOMO $\rightarrow$ LUMO configurations in $S_{1}$. This is a focus of the following discussion. On the contrary, an $i \rightarrow j^{\prime}$ triplet excited configuration is of pure diradical character, and its energy is more determined by the orbital energy gap $\Delta_{i j^{\prime}}$. As a matter of fact, all $T_{2}$ states of the 2B-doped (2N-doped) molecules have the largest contributions from HOMO-1 $\rightarrow$ LUMO $(\mathrm{HOMO} \rightarrow \mathrm{LUMO}+1)$ excitation. The mirror relation on orbital energy changes between 2Band $2 \mathrm{~N}$-dopings results in a mirror relation on the $T_{2}$ characters.

\section{Doped anthracenes with large diradical characters}

All calculated results of the 2B-doped anthracenes' excited states are summarized in Table 1. Figure 3(a) shows that AB1B8, AB2B7, and AB1 $a \mathbf{B} 8 a$ possess exceptionally large diradical characters. The three make the Group III chromophores in Ref. 29. As shown by the results in Table 1, they satisfy Eqs. 1 and 2. The 2B-dopings result in clear diradical Lewis structures in their ground state resonances. Those Lewis structures are shown in 

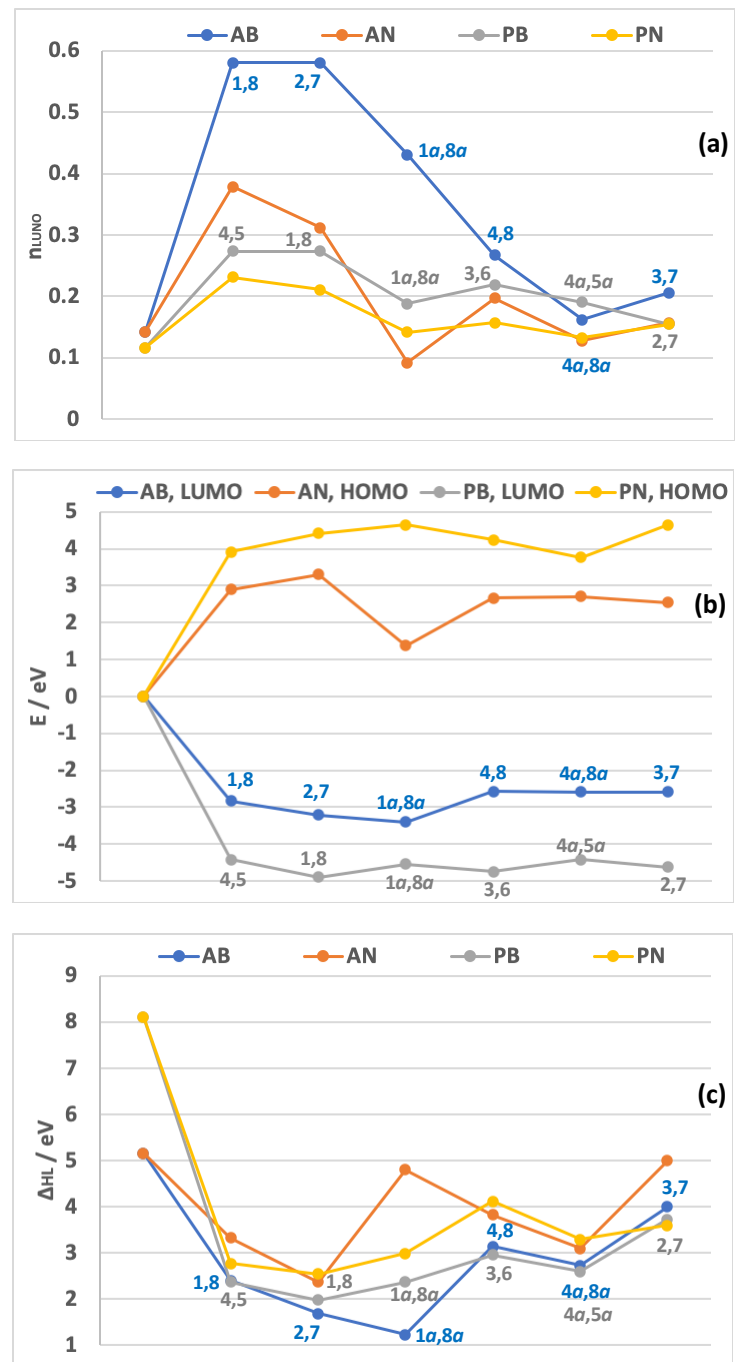

Figure 3: (a) $n_{\mathrm{LUNO}} \mathrm{s}$, (b) changes of molecular orbital energies with respect to undoped molecules, and (c) HOMO-LUMO gaps of all molecules studied in this work. "A" and "P" in the legends indicate doped anthracenes and phenanthrenes. "B" and " $\mathrm{N}$ " indicate 2Band $2 \mathrm{~N}$-dopings. In each panel, the doping positions are given by the $\mathrm{AB}$ and $\mathrm{PB}$ markers, and they are applicable for the corresponding AN and PN markers. The leftmost markers are for the undoped anthracene and phenanthrene.

Figure 4. They are assigned based on natural orbitals of the molecules. For instance, the HONO (highest occupied natural orbital) and LUNO (lowest unoccupied natural orbital) of AB1B8 (Figure S4(a) and (b)) are mainly antisymmetrized and symmetrized combinations of the singly occupied molecular orbitals (SOMOs) of the two allyl moieties on the two terminal rings, pointing to the Lewis structure shown in Figure 4(a). The HONO and LUNO of AB2B7 (Figure S4(e) and (f)) resemble the SOMOs of $m$-quinodimethane (MQDM), and 
Table 1: Calculated quantities for the 2B- and 2N-doped anthracenes discussed in this paper. All energies are given in eV. Excitation energies are relative to $E\left(S_{0}\right)$ of respective molecules.

\begin{tabular}{ccccccccc}
\hline & $E_{v}\left(S_{1}\right)^{a}$ & $E\left(S_{1}\right)$ & $E\left(T_{1}\right)$ & $E\left(T_{2}\right)$ & $f_{S_{1}}$ & $\Delta E_{S F}^{(1)}$ & $\Delta E_{S F}^{(2)}$ & $r_{H L}$ \\
\hline $\mathbf{A}^{b}$ & 3.43 & 3.11 & 1.89 & 3.52 & 0.090 & -0.66 & -0.25 & 28.44 \\
$\mathbf{A B 1 B 8}$ & 1.63 & 1.38 & 0.36 & 1.88 & 0.015 & 0.66 & 1.16 & 0.79 \\
$\mathbf{A B 2 B 7}$ & 0.68 & 0.58 & -0.03 & 1.57 & 0.002 & 0.64 & 1.63 & 5.25 \\
$\mathbf{A B 1} a \mathbf{B 8} a$ & 1.26 & 0.59 & 0.11 & 1.35 & 0.002 & 0.37 & 1.13 & 7.29 \\
$\mathbf{A B} 4 \mathbf{8} 8$ & 2.41 & 2.08 & 0.70 & 2.13 & 0 & 0.68 & 0.73 & 0 \\
$\mathbf{A B 4} a \mathbf{B 8} a$ & 1.76 & 1.22 & 0.80 & 2.20 & 0 & -0.38 & 0.60 & 22.74 \\
$\mathbf{A B 3 B 7}$ & 2.52 & 1.93 & 1.20 & 1.94 & 0 & -0.47 & -0.46 & 0 \\
& & & & & & & & \\
$\mathbf{A N 1 N 8}$ & 2.00 & 1.81 & 0.74 & 1.95 & 0.015 & 0.33 & 0.47 & 0.33 \\
$\mathbf{A N 2 N 7}$ & 1.02 & 0.85 & 0.45 & 1.76 & 0.002 & -0.05 & 0.86 & 4.47 \\
$\mathbf{A N 1} a \mathbf{N} 8 a$ & 1.81 & 1.04 & 0.56 & 1.91 & 0.0004 & -0.08 & 0.79 & 18.98 \\
$\mathbf{A N 4 N 8}$ & 2.56 & 2.18 & 0.93 & 2.10 & 0 & 0.32 & 0.24 & 0 \\
$\mathbf{A N 4} a \mathbf{N} 8 a$ & 1.30 & 0.74 & 0.73 & 2.00 & 0 & -0.72 & 0.54 & 37.97 \\
$\mathbf{A N 3 N 7}$ & 2.79 & 2.17 & 1.40 & 2.24 & 0 & -0.63 & -0.56 & 0 \\
\hline
\end{tabular}

${ }^{a}$ The subscript $v$ indicates vertical $E\left(S_{1}\right)$, which was calculated at the $S_{0}$ structure and with ZPE-correction. ${ }^{b}$ The undoped anthracene.

the corresponding Lewis structure is assigned in Figure 4(c).

The HOMO and LUMO of AB1B8 are shown in Figure S5(b) and (c). The HOMOLUMO gap opening is induced by the bonding interaction between the allyl SOMOs and the empty B $p_{\pi}$ orbitals. A similar situation is seen for AB2B7 (Figure S6(c) and (d)). The gap openings are so small that the two molecules have low-lying $T_{1}$ states. Actually, after the $T_{1}$ geometry optimization, the $T_{1}$ state is found to be the ground state of AB2B7. While both molecules have low $E\left(T_{1}\right)$ s, their $E\left(S_{1}\right)$ s differ by $\sim 1 \mathrm{eV}$. The configuration that contributes the largest portion to the $S_{1}$ state of AB1B8 is HOMO-1 $\rightarrow$ LUMO, followed by $\mathrm{HOMO} \rightarrow \mathrm{LUMO}$, giving a low $r_{H L}=0.79$. The two configurations also make the largest contributions to the $S_{1}$ of $\mathbf{A B 2 B} 7$, but with $r_{H L}=5.25$. While the two sites model is still applicable for AB2B7, it is invalid for AB1B8. This difference is explained using the localized orbitals of the relevant CTs. One of the HOMO-LUMO localized orbitals of AB1B8 


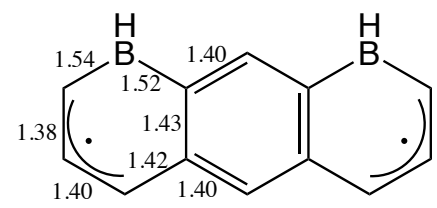

(a) AB1B8

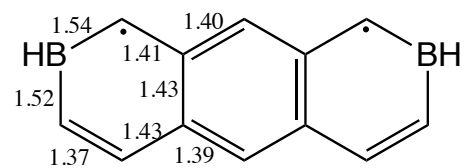

(c) AB2B7

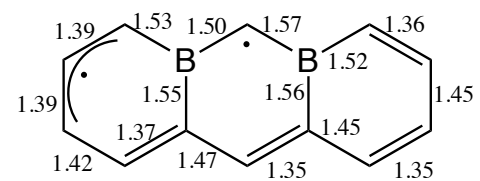

(e) AB1aB8a $S_{0}$

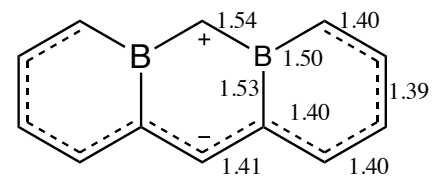

(g) AB1aB8a $S_{1}$

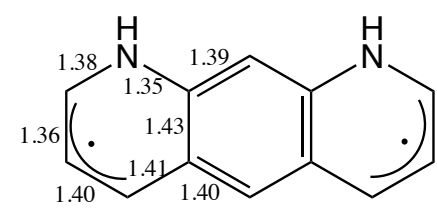

(b) AN1N8

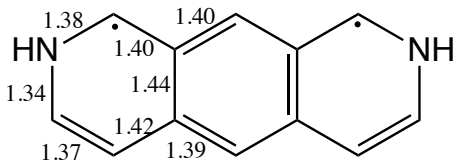

(d) AN2N7<smiles>C1=CB2CC(=Cc3ccccc32)C=C1</smiles>

(f) $\mathrm{AB} 1 \boldsymbol{a B 8} a T_{1}$

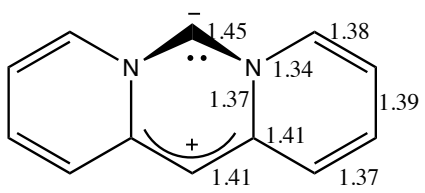

(h) AN1aN8a

Figure 4: Dominant Lewis structures of 2B-doped anthracenes with large diradical characters, and their corresponding $2 \mathrm{~N}$-doped molecules. The bond lengths are given in $\AA$. The Lewis structures and bond lengths are given for ground states of the molecules, unless further specified.

is shown in Figure $\mathrm{S} 5(\mathrm{e})$. The HOMO $\rightarrow$ LUMO excitation corresponds to a CT between this orbital and its symmetry image. This CT can be viewed as between the two allyl fragments in Figure 4(a). It is across the whole molecule along the long axis and needs a high energy $\left(2 K_{H, L}=3.54 \mathrm{eV}\right)$. The HOMO-1-LUMO localized orbitals are highly delocalized along the long axis (Figure S5(f)) and have a much better spatial overlap, substantially lowering the CT energy to $2 K_{H-1, L}=0.96 \mathrm{eV}$. The difference in CT energies compensates the difference between $\Delta_{H, L}$ and $\Delta_{H-1, L}$, so that the HOMO-1 $\rightarrow$ LUMO configuration has a lower energy than the $\mathrm{HOMO} \rightarrow \mathrm{LUMO}$ configuration, and dominates in the $S_{1}$ state.

The HOMO-LUMO and HOMO-1-LUMO localized orbitals of AB2B7 are shown in Figure S6(g) and (h). Compared to the AB1B8 counterpart, the HOMO-LUMO localized orbital is more evenly distributed over the left and central ring. Therefore, the CT between 
this orbital and its symmetry image consists of more portion of short distance transfer between the terminal and the central ring, with $2 K_{H, L}=2.00 \mathrm{eV}$. A lower energy for the $\mathrm{HOMO} \rightarrow \mathrm{LUMO}$ excitation is expected. The orbital in Figure S6(h) is obviously more localized than the one in Figure S6(g), suggesting a slightly higher CT energy $\left(2 K_{H-1, L}=\right.$ $2.06 \mathrm{eV}$ ) for the $\mathrm{HOMO}-1 \rightarrow \mathrm{LUMO}$ excitation. The similar CT energies cannot compensate the difference between $\Delta_{H, L}$ and $\Delta_{H-1, L}$, and therefore, less HOMO-1 $\rightarrow$ LUMO percentage in the $S_{1}$ state of $\mathbf{A B 2 B} 7$ is seen. Although the $S_{1}$ state is dominated by HOMO $\rightarrow \mathrm{LUMO}$, the dominance is way less than in the $S_{1}$ state of anthracene $\left(r_{H L}=5.25\right.$ vs. 28.44). This is a reflection of the $\Delta_{H-1, L}$ reduction on the $2 \mathrm{~B}$-doping.

The transition dipole moments between the molecular orbitals are substantial: 3.83 and 1.52 a.u. for the HOMO-LUMO and HOMO-1-LUMO pairs of AB1B8; 2.35 and 2.42 a.u. for the corresponding pairs of AB2B7. However, there is a significant destructive interference of those transition dipoles in the mixing of the HOMO $\rightarrow$ LUMO and HOMO-1 $\rightarrow$ LUMO configurations, resulting in the small $f_{S_{1}}=0.015$ and 0.002 of the two molecules. The destructive interference is rooted in the minimization of the CT energy in the $S_{0}-S_{1}$ excitation. Shown in Figure S7(a) and (b) are the HONO and LUNO of the $S_{1}$ state of AB1B8. Their close-to-1 occupancies indicate a good one-electron excitation scheme. The mixing of $\mathrm{HOMO} \rightarrow \mathrm{LUMO}$ and $\mathrm{HOMO}-1 \rightarrow$ LUMO configurations is essentially to mix the HOMO and HOMO-1 to result in a hole orbital that resembles the HONO. Clearly, the HONO and LUNO have their amplitudes on different sets of atoms, and their $2 K_{\text {HONO,LUNO }}$ is minimized to $0.31 \mathrm{eV}$. The CT energy in the $S_{0^{-}} S_{1}$ excitation has thus been minimized. This mixing occurs whenever the CT energy lowering overcomes the cost of the larger orbital energy gap $\Delta_{H-1, L}$ involved in the excitation. The LUNO resembles the LUMO. The LUMO has a less tendency to mix with the LUMO+1 to achieve the lower CT energy than the HOMO-1-HOMO mixing because $\Delta_{L, L+1}>\Delta_{H-1, H}$. Now with the hole and particle orbitals (HONO and LUNO) on different sets of atoms, their transition dipole integral must be small, explaining the small $f_{S_{1}}=0.015$. 
It is interesting to inspect the $T_{1} \mathrm{HONO}$ and LUNO, which are shown in Figure S7(c) and (d). They resemble more the HOMO and LUMO. There is some discernible difference between the HONO and HOMO (Figure S5(b)), which arises from mixing a small portion of the HOMO-1 to the HOMO so that the amplitudes on $\mathrm{C} 1 a$ and $\mathrm{C} 8 a$ are reduced and those on $\mathrm{C} 2$ and $\mathrm{C} 7$ are enhanced. The purpose is to enhance the spatial overlap of the hole and particle orbitals and thus increase the $2 K_{\text {HONO,LUNO }}$ to $5.02 \mathrm{eV}$, since this exchange integral is a stabilizing factor for the triplet excitation. The arguments in these two paragraphs also apply to AB2B7, whose $S_{1}$ and $T_{1}$ HONOs and LUNOs are shown in Figure S7(e)-(h).

The $S_{0}$ state of $\mathbf{A B 1} a \mathbf{B 8} a$ underwent a distortion to a $C_{s}$ planar structure during geometry optimization. The 2B-doping isolates two odd-numbered C fragments, C9 and the leftover 11-membered $\mathrm{C}$ chain. The distortion concentrates the radical center on one side of the $\mathrm{C}$ chain, which interacts more effectively with the $\mathrm{C} 9$ radical through $\mathrm{B} 8 \mathrm{a}$. The bond lengths (Figure 4(e)) indicate that it is an allyl moiety that contains the radical center on the $\mathrm{C}_{11}$ chain, which is corroborated by the HONO and LUNO (Figure S4(i) and (j)). We assigned the Lewis structure in Figure 4(e). The molecule has the smallest $\Delta_{H, L}=1.22 \mathrm{eV}$ among all studied molecules. The $\mathrm{HOMO}-1$ to $\mathrm{LUMO}+1$, and the relevant localized orbitals are shown in Figure S8(a)-(d). The HOMO-LUMO localized orbitals (Figure S8(e) and (f)) are concentrated at the central ring, with small extensions on the side rings. With their good spatial overlap, the CT energy of HOMO $\rightarrow$ LUMO is relatively low $\left(2 K_{H, L}=2.02 \mathrm{eV}\right)$. The HOMO-1-LUMO localized orbitals are shown in Figure S8(g) and (h), which also suggest a short distance $\mathrm{CT}\left(2 K_{H-1, L}=1.64 \mathrm{eV}\right)$. The not much larger $2 K_{H, L}$ and the small $\Delta_{H, L}$ are responsible for the dominance of $\mathrm{HOMO} \rightarrow \mathrm{LUMO}$ in $S_{1}$, with $r_{H L}=7.29$. The HOMO-1-LUMO configuration makes the second largest contribution to $S_{1}$. The HOMOLUMO transition dipole moment has a small magnitude of $0.99 \mathrm{a} . \mathrm{u}$, and it has a destructive interference with the 2.48 a.u. large HOMO-1-LUMO transition dipole, resulting in the small $f_{S 1}=0.002$.

Both the $S_{1}$ and $T_{1}$ states reorganize to $C_{2 v}$ structures, with 0.67 and $0.27 \mathrm{eV}$ reorgani- 
zation energies, respectively. The two SOMOs of the $T_{1}$ state are shown in Figure S8(i) and (j). They resemble the HOMO-LUMO localized orbitals in Figure S8(e) and (f), as expected. The SOMOs do not interact in $T_{1}$, and therefore there is no tendency to distort the structure to concentrate them on one side of the molecule and enhance their interaction, as in the $S_{0}$ state. Actually, the $S_{0} \mathrm{HOMO}$ and LUMO clearly arise from the bonding and antibonding mixings of the the SOMOs of $A_{2}$ and $B_{2}$ IRREPs, respectively, which is turned on by the pseudo-Jahn-Teller distortion ${ }^{55}$ along a $b_{1}$ mode from $C_{2 v}$ to $C_{s}$. The $S_{1}$ state at the optimized structure features a CT from the SOMO in Figure S8(j) to the SOMO in Figure S8(i), corresponding to the Lewis structure in Figure 4(e). Compared to the $S_{0}$ structure, the $S_{1}$ state features a bond length equalization of the undecapentenyl anion fragment, and thus the $0.67 \mathrm{eV}$ large reorganization energy.

While all the three 2B-doped anthracenes have large positive $\Delta_{S F}^{(1)} \mathrm{s}$ and $\Delta_{S F}^{(2)} \mathrm{s}$, their too low $E\left(T_{1}\right) \mathrm{s}$ imply instabilities. Also, not many acceptors can harvest the low-lying $T_{1}$ excitons, and the low energy excitons tend to lead to a low open-circuit voltage. ${ }^{56}$ The $2 \mathrm{~B}-$ doped anthracenes are hence not good SF chromophore candidates. The $K_{a, b} \mathrm{~s}$ of the three 2B-doped molecules are larger than that of anthracene (Figure 5(a)). When we remove the B atoms, the leftover $\mathrm{C}$ fragments are all diradicals with ferromagnetic couplings, and therefore their SOMOs are not AH paired, resulting in nonnegligible exchange integrals between each pair of the SOMOs. These integrals are carried onto the 2B-doped molecules, where the SOMOs become HOMOs and LUMOs. The large $K_{a, b}=0.56$ and $0.66 \mathrm{eV}$ of AB2B7 and AB1 $a$ B8 $a$ contribute to their exceptionally low $E\left(T_{1}\right)$ s. The unpaired electrons densities of AB1B8, AB2B7, and $\mathbf{A B 1} a \mathbf{B 8} a$ are shown in Figure S9. They are consistent with the diradical Lewis structures in Figure 4. Since the unpaired electrons densities deliver the same information as the HONO and LUNO, they are not shown for the molecules discussed below.

Figure 3(a) shows that all 2N-doped molecules have lower diradical characters than their 2B-doped analogues. This is consistent with the larger $\Delta_{H, L} \mathrm{~S}$ of all $2 \mathrm{~N}$-doped molecules 


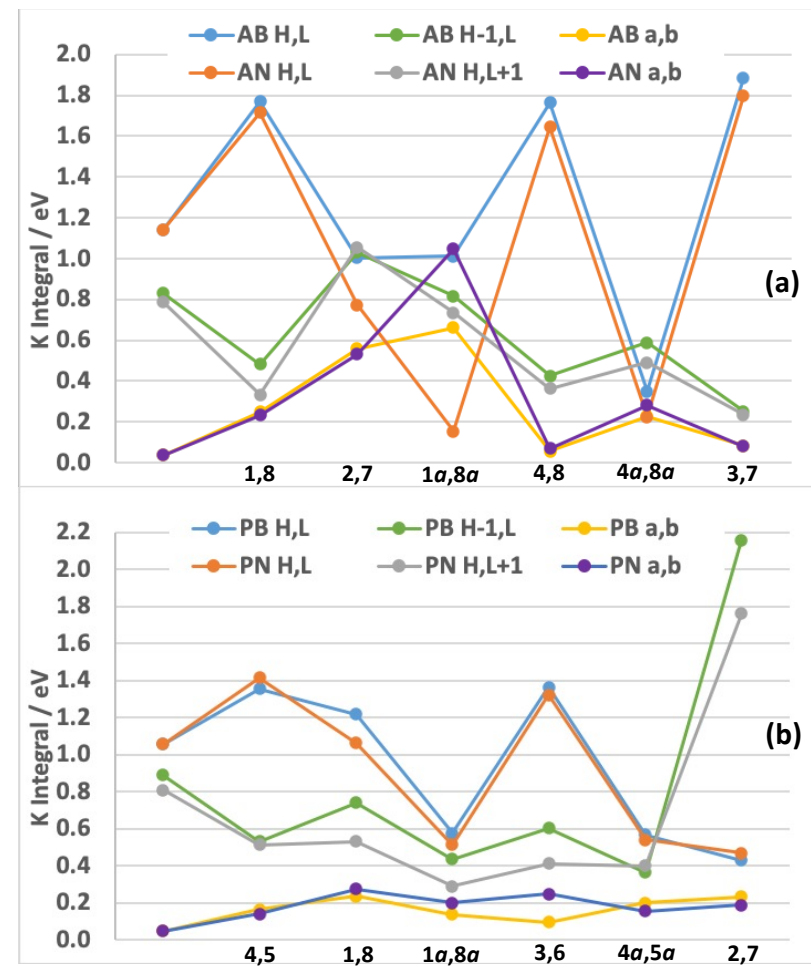

Figure 5: The calculated exchange integrals for canonical orbitals and localized orbitals of the 2B- and 2N-doped anthracenes (a) and phenanthrenes (b). The doping positions are shown at the bottom of each panel, beneath the corresponding markers. The first markers on the left are for the undoped molecules. The "H,L", "a,b" etc. in the legends denote the subscripts of the $K$ integrals. For PB2B7, the integrals are for natural orbitals and their localized orbitals (see the relevant discussion in text).

as shown in Figure $3(\mathrm{c})$. The $K_{H, L} s$ of the $2 \mathrm{~N}$-doped molecules, except $\mathbf{A B} \mathbf{1} a \mathbf{B} 8 a$, are close to the 2B analogues (Figure 5(a) and (b)). With the smaller diradical characters, the $2 \mathrm{~N}$-doped molecules are anticipated to have higher $E\left(T_{1}\right) \mathrm{s}$. However, it is necessary to point out that this anticipation is not always met, especially after considering possibly different $T_{1}$ reorganization energies of the $2 \mathrm{~B}$ - and $2 \mathrm{~N}$-doped molecules and their different $K_{a, b} \mathrm{~s} . \mathbf{A N 1 N 8}, \mathbf{A N 2 N 7}$, and AN1 $a \mathbf{N 8} a$ have their $E\left(T_{1}\right)$ s higher by $\sim 0.4 \mathrm{eV}$ than their 2B analogues. Their $E\left(S_{1}\right)$ s are also higher. All three have $\Delta_{S F}^{(2)}>0$, and $\Delta_{S F}^{(1)}>0$ or just slightly negative. Of special interest is $\mathbf{A N 1 N 8}$, whose $E\left(T_{1}\right)$ is $0.74 \mathrm{eV}$. It is not much lower than the $0.86 \mathrm{eV} E\left(T_{1}\right)$ of pentacene, which is the most intensely investigated SF chromophore.

Like AB1B8, the $S_{1}$ state of AN1N8 is not dominated by the HOMO $\rightarrow$ LUMO config- 
uration, but by the $\mathrm{HOMO} \rightarrow \mathrm{LUMO}+1$ configuration, with $r_{H L}=0.33$. The mirror relation holds between AB1B8 and AN1N8, and as shown below, for most pairs of 2B- and 2Ndopings. The correlations between the canonical orbitals of AB1B8 and AN1N8 are shown in Figure S5(m). The HOMO and LUMO of AN1N8 (Figure S5(h) and (i)) correlate to the LUMO and HOMO of AB1B8 (Figure S5(c) and (b)). The bonding interaction with B in the AB1B8 HOMO becomes antibonding interaction with $\mathrm{N}$ in AN1N8. This is another mirror relation anticipated based on the acceptor and donor characters of the two dopants and the first order perturbation theory. Overall, the dopings can be viewed as introducing perturbations to the $\pi$ system of the leftover $\mathrm{C}_{12}$ system. Bonding perturbations of introducing acceptors must correspond to antibonding perturbations of introducing donors. The nonbonding LUMO of AB1B8 remains nonbonding in AN1N8 and thus becomes HOMO. Given the similar HOMO-LUMO pairs, the HOMO-LUMO localized orbitals of AN1N8 looks similar to those of AB1B8 (Figure S5(e) vs. (k)), except replacing the B-C bonding by N-C antibonding. A similar long distance and high energy CT $\left(2 K_{H L}=3.42 \mathrm{eV}\right)$ between the two terminal rings occurs in the $\mathrm{HOMO} \rightarrow$ LUMO excitation, diminishing its percentage in $S_{1}$ of $\mathrm{AN} 1 \mathrm{~N} 8$.

The HOMO-LUMO+1 localized orbital is shown in Figure S5(1), and it is evenly distributed across the molecule, just like the HOMO-1-LUMO localized orbital of AB1B8 (Figure S5(f)). The obvious topological similarity between the two localized orbitals is not accidental. In both molecules, the $\mathrm{HOMO}-1$ and $\mathrm{LUMO}+1$ orbitals are not perturbed by the dopants since they have antisymmetric phases around each dopant (Figure S5(a),(d),(g),(i)). Therefore, there are correlations between the HOMO-1 orbitals and between the LUMO-1 orbitals in terms of their C-C bonding patterns. Within each molecule, the HOMO-1 and LUMO+1 orbitals are paired by AH symmetry. The correlations and pairings together determine that the HOMO-1 of AB1B8 and the LUMO+1 of $\mathbf{A N 1 N 8}$ are distributed on the same set of $\mathrm{C}$ atoms, and can be viewed as a bonding-antibonding pair (Figure S5(a) vs. (j)). Together with the correlation between the HOMO of AN1N8 and the LUMO of 


\begin{abstract}
AB1B8, the HOMO-LUMO+1 localized orbitals of the former molecule must spread over the same set of atoms as the HOMO-1-LUMO localized orbitals of the latter molecule, along with the opposite bonding/antibonding characters between adjacent atoms (Figure S5(f) vs. (l)). A short distance low energy CT $\left(2 K_{H, L+1}=0.66 \mathrm{eV}\right)$ is hence expected for the
\end{abstract} $\mathrm{HOMO} \rightarrow \mathrm{LUMO}+1$ excitation in AN1N8, similar to the HOMO-1 $\rightarrow$ LUMO excitation in AB1B8. Consequently, the $\mathrm{HOMO} \rightarrow \mathrm{LUMO}+1$ excitation dominates the $S_{1}$ state of AN1N8.

Overall, the mirror relation between the $S_{1}$ states' compositions of AB1B8 and AN1N8 is a result of the following: (1) the mirror relation between LUMO energy decrease of the 2Bdoping and HOMO energy increase of the 2N-doping; (2) the HOMO-to-LUMO and LUMOto-HOMO correlations between the two molecules; (3) the AH pairing of doubly occupied and unoccupied orbitals of the $\mathrm{C}_{12}$ fragment. All these apply to the AB2B7-AN2N7 pair. Therefore, the $S_{1}$ of AN2N7 is dominated by HOMO $\rightarrow$ LUMO, and supplemented by $\mathrm{HOMO} \rightarrow \mathrm{LUMO}+1$, with $r_{H L}=4.47$. The orbital correlation diagram between AB2B7 and AN2N7 appears to be different from that between AB1B8 and AN1N8 (Figure S6(q) vs. Figure S5(m)). But the differences in the C-C bonding pattern correlations are counterpoised by the differences in the AH pairing correlations. Therefore, the HOMO-1-LUMO localized orbitals of AB2B7 and the HOMO-LUMO+1 localized orbitals of AN2N7 are similarly correlated as the localized orbitals of AB1B8 and AN1N8: (1) B-C bonding is replaced by $\mathrm{N}-\mathrm{C}$ antibonding; (2) C-C bonding (antibonding) is replaced by $\mathrm{C}-\mathrm{C}$ antibonding (bonding).

As mentioned above, the mixings of the HOMO-1 $\rightarrow$ LUMO and HOMO $\rightarrow$ LUMO configurations and their destructive interferences of transition dipoles in the $S_{1}$ states of AB1B8 and AB2B7 are driven by the hole orbital polarizations that minimize the CT energies in the $S_{0}-S_{1}$ excitation. A similar argument applies to the HOMO $\rightarrow$ LUMO and $\mathrm{HOMO} \rightarrow \mathrm{LUMO}+1$ configurations in $\mathbf{A N 1 N 8}$ and $\mathbf{A N 2 N 7}$, but instead with the particle orbital polarizations realized by mixing LUMO and LUMO+1. The hole and particle orbitals (HONOs and LUNOs) of the $S_{1}$ state of AN1N8 are shown in Figure S7(i) and (j). The 
LUNO is obviously a mixture of LUMO and LUMO+1. With the mixture, the LUNO and HONO have amplitudes on different sets of atoms (as much as possible), so that both the CT energy and transition dipole are reduced. The $f_{S_{1}}$ s of AN1N8 and AN2N7 are hence as small as those of the $2 \mathrm{~B}$ counterparts.

As an exception to the caption of this section, $\mathbf{A N} 1 a \mathbf{N} 8 a$ has the lowest diradical character among all studied molecules (Figure 3(a)). The molecule is placed in this section to be compared with $\mathbf{A B} \mathbf{1} a \mathbf{B} 8 a$. The mirror relation in orbital energy changes does not hold for the AB1 $a \mathbf{B} 8 a-\mathbf{A N} 1 a \mathbf{N} 8 a$ pair (Figure $3(\mathrm{~b})$ ). The $\Delta_{H, L}$ of $\mathbf{A N 1} a \mathbf{N} 8 a$ is exceptionally large (Figure 3(c)). The molecule possess a $C_{s}$ puckered $S_{0}$ structure. The HOMO and LUMO are shown in Figure S8(l) and (m). They correlate with the LOMO and HOMO of the $S_{1}$ state of $\mathbf{A B 1} a \mathbf{B} 8 a$ (Figure $\mathrm{S} 8(\mathrm{j})$ and (i)). Therefore, a similar ionic Lewis structure is assigned to AN1 $a \mathbf{N 8}$ a (Figure 4(h)), but with opposite charge localizations. The two butadienyl fragments are assigned to the two sides based on the HONO-1 to LUNO+1 (Figure $\mathrm{S} 4(\mathrm{k})-(\mathrm{n})$ ), which are symmetrized and antisymmetrized combinations of HONOs and LUNOs of the two butadienyls. The nonplanar structure reduces the conjugation between the terminal and the central ring, resulting in the localized $\pi$ systems. C9 is sandwiched by two electronegative $\mathrm{N}$ atoms and is thus capable of accommodating another electron. The cramming of six electrons in the N-C9-N $\pi$ system maximizes the $\pi$ antibonding interaction among the atoms. A pyramidalization of $\mathrm{C} 9$ ensues. The low adiabatic $E\left(S_{1}\right)=0.59 \mathrm{eV}$ of $\mathrm{AB} 1 a \mathrm{~B} 8 a$ hints at the possibility of swapping diradical and ionic characters of $S_{0}$ and $S_{1}$, which is indeed the case in $\mathbf{A N 1} a \mathbf{N 8} a$. The strong ionic character of AN1 $a$ N8 $a$ explains its low diradical character. The different canonical orbitals also lead to the different exchange integrals between this pair of 2B- and 2N-doping in Figure 5(a).

The low diradical character is not incompatible with a low $E\left(T_{1}\right)$. The antibonding interaction within the $\mathrm{N}-\mathrm{C} 9-\mathrm{N}$ moiety is diminished in the HOMO $\rightarrow$ LUMO excitation, so that both $S_{1}$ and $T_{1}$ undergo reorganization back to $C_{2 v}$ planar structure. The associated $0.95 \mathrm{eV}$ large reorganization energy lowers $E\left(T_{1}\right)$ to $0.56 \mathrm{eV}$. The $S_{1}$ reorganization energy 


\section{Doped anthracenes with intermediate diradical characters}

The other three 2B-doped anthracenes, AB4B8, AB4aB8a, and AB3B7, possess far less diradical characters (Figure 3(a)). Correspondingly, their $E\left(T_{1}\right) \mathrm{s}$ are higher. These molecules are of $C_{2 h}$ symmetry. They belong to Group II chromophores classified by Stoycheva et al. ${ }^{29}$ Among them, AB4B8 has the largest diradical character and is the only one that satisfies both Eqs. 1 and 2. The HONO and LUNO (Figure S10(a) and (b)) of the molecule show clear characters of $p$-quinodipropane (PQDP), with the two allyls at the two terminal rings interacting with the central phenyl. The corresponding Lewis structure is assigned in Figure 6(a). The canonical orbitals and their localized orbitals are shown in Figure S11(a)-(i). As expected, the HOMO-LUMO localized orbitals (Figure $\mathrm{S} 11(\mathrm{~g}))$ are concentrated on the terminal allyls that are separated along the diagonal direction of the molecular plane. The localization of the orbitals is well reflected by the small $K_{a, b}=0.06 \mathrm{eV}$. A long distance $\mathrm{CT}$ is involved in the HOMO $\rightarrow$ LUMO excitation 
$\left(2 K_{H, L}=3.52 \mathrm{eV}\right)$. The HOMO-1-LUMO localized orbitals are shown in Figure S11(h) and (i) and exhibit a better spatial overlap. A short distance CT $\left(2 K_{H-1, L}=0.84 \mathrm{eV}\right)$ is involved in the HOMO-1 $\rightarrow$ LUMO excitation. The $S_{1}$ state is hence dominated by HOMO-1 $\rightarrow$ LUMO. Also, since the HOMO-1 $\rightarrow$ LUMO and HOMO $\rightarrow$ LUMO configurations are of $A_{g}$ and $B_{u}$ IRREPs, respectively, they do not interact and mix. The $S_{1}$ state does not contain $\mathrm{HOMO} \rightarrow \mathrm{LUMO}$, and because of symmetry, it is a dark state with $f_{S_{1}}=0$. The HOMO $\rightarrow$ LUMO configuration makes the largest contribution to $S_{2}$, with vertical $E\left(S_{2}\right)=2.92 \mathrm{eV}$ and $f_{S_{2}}=0.196$.

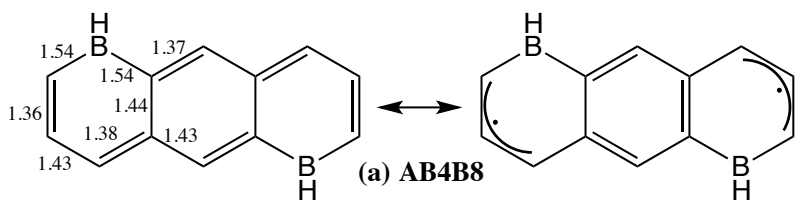

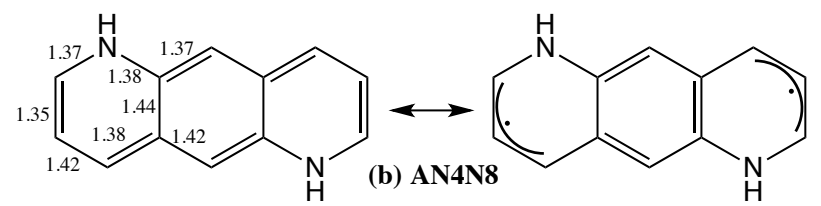

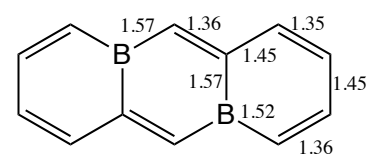

(c) $\mathrm{AB} 4 a \mathrm{B8} a$

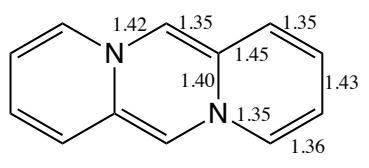

(d) $\mathrm{AN} 4 a \mathrm{~N} 8 a$

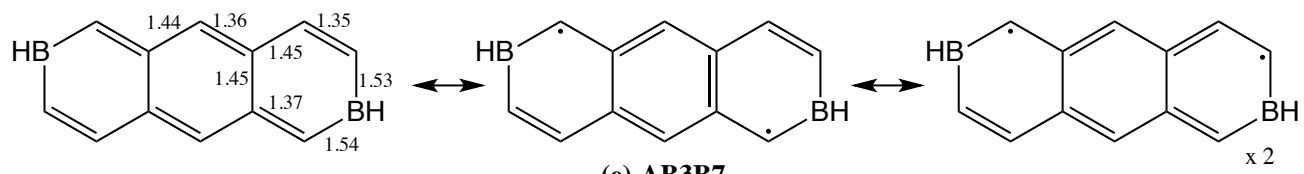

(e) $\mathrm{AB3B} 7$

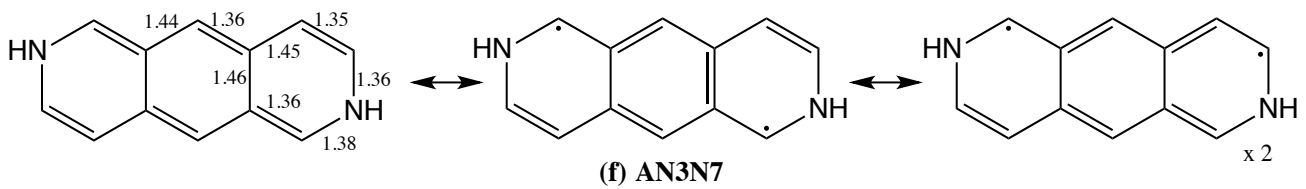

Figure 6: Dominant Lewis structures of 2B-doped anthracenes with intermediate diradical characters, and their corresponding $2 \mathrm{~N}$-doped molecules. The bond lengths are given in $\AA$. The Lewis structures and bond lengths are given for ground states of the molecules. The " $\times 2$ " in rightmost Lewis structures in (e) and (f) indicate that there are two such structures that are symmetry images of each other.

It is interesting to compare $\mathbf{A B} 4 \mathbf{B} 8$ and $\mathbf{A B 1 B 8}$, whose $\mathrm{C}_{12}$ fragments are PQDP and 
MQDP, respectively. According to Ovchinnikov's rule, ${ }^{57}$ MQDP has a ferromagnetic coupling of the two radical centers, while PQDP has an anti-ferromagnetic coupling and hence AB4B8 possesses a lower diradical character. ${ }^{29}$ Also, the HOMO and LUMO of AB4B8 are AH paired, while those of AB1B8 are not. The similar terminal allyls Lewis structures lead to long distance $\mathrm{CT}$ between the terminal rings in the respective $\mathrm{HOMO} \rightarrow \mathrm{LUMO}$ excitations. Therefore, both molecules have their $S_{1}$ states being dominated by HOMO-1 $\rightarrow$ LUMO excitation. Because of their different symmetries, the AB1B8 $S_{1}$ consists of HOMO $\rightarrow$ LUMO configuration, which is absent in the AB4B8 $S_{1}$. The hole orbital polarization through mixing $\mathrm{HOMO}-1$ and $\mathrm{HOMO}$ does not occur in AB4B8's $S_{1}$ state because of the different IRREPs of the canonical orbitals. AB4B8 has a not too low $E\left(T_{1}\right)$ and its vertical absorption to $S_{2}$ occurs at the blue region of solar spectrum with fairly high irradiance. ${ }^{58}$ It is a candidate of SF chromophore.

The natural orbitals (Figure S12(a)-(d)) and bond lengths of AB4aB8a indicate a Lewis structure with two hexatrienyl fragments (Figure $6(\mathrm{c})$ ). The pseudo-degenerate $n_{\mathrm{HONO}-1}=$ 1.88 and $n_{\mathrm{HONO}}=1.86$ suggest that the localized orbitals of the HONO-1-HONO pair reflect the ground state wave function to a similar extent. They (Figure S12)(e) and (f)) show characters of the HONOs of the two hexatrienyls. The pseudo-degenerate $n_{\mathrm{LUNO}}=0.16$ and $n_{\mathrm{LUNO}+1}=0.13$ likewise allow us to use the localized orbitals of the LUNO-LUNO+1 pair to view the ground state, and they (Figure S12)(g) and (h)) resemble the two LUNOs of the hexatrienyls. The short polyenyl fragments feature low diradical character, speaking for the smallest $n_{\text {LUNO }}$ among the 2B-doped anthracenes. The canonical orbitals and their localized orbitals of the molecule are shown in Figure S13(a)-(g). The HOMO-LUMO localized orbitals show a better spatial overlap than those of the HOMO-1-LUMO pair $\left(2 K_{H, L}=0.70 \mathrm{eV}\right.$ vs. $\left.2 K_{H-1, L}=1.18 \mathrm{eV}\right)$. Naturally, the $S_{1}$ state is dominated by HOMO $\rightarrow$ LUMO, with $r_{H L}=22.74$. It is a dark state because of symmetry. The short distance CT explains the low $E\left(S_{1}\right)$ and the failure to satisfy Eq. 1.

The HONO and LUNO of AB3B7 (Figure S14(b) and (c)) and the bond lengths indicate 
the Lewis structure in Figure 6(e). The diradical character of this molecule is associated to the central p-quinodimethane (PQDM) fragment. The HONO and HONO-1 are pseudodegenerate $\left(n_{\mathrm{HONO}-1}=1.88\right.$ and $\left.n_{\mathrm{HONO}}=1.82\right)$. Their localized orbitals (Figure S14(e)) resemble the HONOs of the two hexatrienyls in Figure 6(e). The LUNO and LUNO+1 are less pseudo-degenerate $\left(n_{\mathrm{LUNO}}=0.21\right.$ and $\left.n_{\mathrm{LUNO}+1}=0.12\right)$. Their localized orbitals (Figure S14(f)) resemble LUNOs of the hexatrienyls. The localized natural orbitals indicate multiple diradical characters in addition to PQDM, i.e., those involving the hexatrienyl diradical structures.

The relation between AB4B8 and AB3B7 is similar to the relation between AB1B8 and AB2B7. Therefore, AB3B7 shares a few common features with AB4B8, $r_{H L}=0$, and an $S_{1}$ dominated by HOMO-1 $\rightarrow$ LUMO being dark because of symmetry. However, the AB3B7 does not satisfy Eq. 1, a result of its smaller diradical character. Shorter exocyclic odd-numbered $\mathrm{C}$ fragments at $p$ positions have larger SOMO lobes in contact with the phenyl frontier orbitals, leading to a stronger anti-ferromagnetic coupling and lower diradical character. Correspondingly, the $\Delta_{H, L}$ of AB3B7 is larger than the AB4B8 counterpart (3.99 vs $3.13 \mathrm{eV}$ ), while their $K_{H, L} \mathrm{~S}$ are similar $(1.88 \mathrm{vs} 1.76 \mathrm{eV}$ ). The diradical character of the former is hence smaller. This molecule features a low $E\left(T_{2}\right)$ and does not satisfy Eq. 2 either. As mentioned above, this is attributed to the multiple diradical characters of PQDM + hexatrienyls. The pseudo-degeneracy between HONO-1 and HONO is associated with the $0.64 \mathrm{eV}$ small $\Delta_{H-1, H}$ (Figure $2(\mathrm{a})$ ), which is responsible for the low-lying $T_{2}$ characterized by HOMO $-1 \rightarrow$ LUMO excitation. It is necessary to point out the difference between multiple diradical characters and tetraradical character. A substantial tetraradical character in the ground state of a molecule can lead to a low-lying $T_{2}$ state with the same tetraradical character, ${ }^{15}$ which is disadvantageous for satisfying Eq. 2. In the multiple diradical characters case, the low-lying $T_{2}$ is still a diradical state.

The mirror relations hold for the 2N-doped counterparts. AN4N8, AN4aN8a, and AN3N7 share similar natural orbitals (Figures S10, S12, S14) and adopt similar Lewis 
structures as the 2B analogues (Figure 6(b),(d),(f)). The 2N-doped molecules have similar $S_{1}$ characters as the $2 \mathrm{~B}$ species, except that the roles of $\mathrm{HOMO}-1 \rightarrow \mathrm{LUMO}$ in the $2 \mathrm{~B}$ analogues are taken by $\mathrm{HOMO} \rightarrow \mathrm{LUMO}+1$. Note that the small $\Delta_{H-1, H}$ of $\mathbf{A B} 3 \mathbf{B} 7$ is translated to the small $\Delta_{L, L+1}$ of AN3N7 (Figure 2(a) and (b)), which also features multiple diradical characters, and consequently a low-lying $T_{2}$ and the failure to satisfy Eq. 2. The topological correlations between frontier canonical orbitals of the 2B- and 2N-doped molecules are given in Figures S11(s), S13(o), and S15(o). These correlations underly good parallel variations of the key exchange integrals (Figure 5(a)) and the mirror relations between the two sets of molecules. AN4N8 is the only one among the three that satisfy Eqs. 1 and 2. The generally smaller diradical character induced by 2N-doping gives AN4N8 a higher $E\left(T_{1}\right)=0.93 \mathrm{eV}$, which is beneficial for harvesting $T_{1}$ excitons and high open-circuit voltage.

\section{B-doped phenanthrenes}

All the studied 2B-doped phenanthrenes have relatively mild diradical characters (Figure 3(a)). Yet, five of the six (except PB2B7) have low enough $E\left(T_{1}\right)$ s to satisfy Eq. 1. All the calculated results for the excited states of the $2 \mathrm{~B}$ - and $2 \mathrm{~N}$-doped phenanthrenes are summarized in Table 2. The detailed analyses of the electronic structures of the 2B-doped phenanthrenes are given in SI (Section S3). Similar factors determine the $S_{1}$ compositions and the applicability of the two sites model: $\Delta_{H-1, H}, K_{H, L}$ vs. $K_{H-1, L}$, and hole orbital polarization. The hole orbital polarization underlies small $f_{S_{1}}$. Regardless of the applicability of the two sites model, large diradical character and $K_{a, b}$ favor low $E\left(T_{1}\right)$ and the satisfaction of Eq. 1. The different hole particle polarizations in $S_{1}$ and $T_{1}$ can explain the different reorganization energies of the two states. The satisfaction of Eq. 1 can be reached by the larger $T_{1}$ reorganization energy. Small $\Delta_{H-1, H}$ may result in multiple diradical character, which speaks for low $E\left(T_{2}\right)$, and the consequent possible crossing of $T_{1}$ and $T_{2} \mathrm{PESs}$, as well as the propensity of not satisfying Eq. 2. $\Delta_{H-1, H} \approx 0$ also leads to ambiguity of 
$r_{H L}$. For such cases, it is more reasonable to use $r_{H O N O, L U N O}$ to characterize $S_{1}$. Among the 2B-doped phenanthrenes that satisfy Eqs. 1 and 2, PB1aB8a is the best SF chromophore candidate, as it has the highest $0.62 \mathrm{eV} E\left(T_{1}\right)$. The disadvantage of its 0.018 low $f_{S_{1}}$ can be counterpoised by the high irradiance at the $\sim 1.90 \mathrm{eV}$ (vertical $E\left(S_{1}\right)$ ) region of the solar spectrum.

Table 2: Calculated quantities for the 2B- and 2N-doped phenanthrenes discussed in this paper. All energies are given in eV. Excitation energies are relative to $E\left(S_{0}\right)$ of respective molecules.

\begin{tabular}{ccccccccc}
\hline & $E_{v}\left(S_{1}\right)^{a}$ & $E\left(S_{1}\right)$ & $E\left(T_{1}\right)$ & $E\left(T_{2}\right)$ & $f_{S_{1}}$ & $\Delta E_{S F}^{(1)}$ & $\Delta E_{S F}^{(2)}$ & $r_{H L}$ \\
\hline $\mathbf{P}^{b}$ & 3.98 & 3.81 & 2.82 & 3.60 & 0.0003 & -1.83 & -2.03 & 0 \\
PB4B5 & 1.78 & 1.50 & 0.34 & 2.09 & 0.054 & 0.82 & 1.41 & 6.61 \\
PB1B8 & 1.87 & 1.59 & 0.37 & 2.04 & 0.067 & 0.85 & 1.30 & 4.33 \\
PB1 $a$ B8 $a$ & 1.90 & 1.41 & 0.62 & 1.80 & 0.018 & 0.17 & 0.56 & 11.86 \\
PB3B6 & 2.51 & 2.03 & 0.86 & $1.80^{c}$ & 0.009 & 0.31 & 0.08 & 0.48 \\
PB4aB5 $a$ & 1.37 & 1.18 & 0.50 & 1.66 & 0.029 & 0.18 & 0.66 & 14.06 \\
PB2B7 & 2.37 & 1.94 & 1.05 & 1.73 & 0.004 & -0.16 & -0.37 & $3.8^{d}$ \\
& & & & & & & & \\
PN4N5 & 1.96 & 1.44 & 0.49 & 2.13 & 0.061 & 0.46 & 1.15 & 7.84 \\
PN1N8 & 1.39 & 1.04 & 0.23 & 1.89 & 0.044 & 0.58 & 1.43 & 9.32 \\
PN1 $a N 8 a$ & 1.32 & 0.89 & 0.46 & 1.48 & 0.006 & -0.03 & 0.56 & 9.00 \\
PN3N6 & 1.62 & 0.96 & 0.16 & 0.76 & 0.002 & 0.64 & 0.44 & 0.10 \\
PN4 $a$ N5 $a$ & 1.53 & 1.12 & 0.61 & 1.85 & 0.020 & -0.10 & 0.63 & 14.53 \\
PN2N7 & 2.38 & 1.93 & 1.10 & 1.99 & 0.002 & -0.27 & -0.21 & 2.97 \\
\hline
\end{tabular}

${ }^{a}$ The subscript $v$ indicates vertical $E\left(S_{1}\right)$, which was calculated at the $S_{0}$ structure and with ZPE-correction. ${ }^{b}$ The undoped phenanthrene. ${ }^{c}$ This is the $E\left(T_{2}\right)$ calculated at the $T_{1}$ structure. ${ }^{d}$ This $r_{H L}$ is for the $S_{1}$ wave function expressed using the natural orbitals of the state.

\section{N-doped phenanthrenes}

Four (except PN1N8 and PN1 $a$ N8a) of the six investigated 2N-doped phenanthrenes undergo pyramidalizations at their $\mathrm{N}$ centers and distort their structures to $C_{2}$ symmetry. This is different from the 2B-doped analogues, but not unexpected, since the doping of $\mathrm{N}$ atoms 
comes with filling antibonding orbitals of phenanthrene. PN4aN5a has the most severe distortion because of the adjacency of the two $\mathrm{N}$ lone pairs. The C1a-N4a-N5a-C8a dihedral angle is $41^{\circ}$ large. In general, none of the $2 \mathrm{~N}$-doped phenanthrenes are good SF chromophore candidates. PN4aN5a is the only one that has not too low $E\left(T_{1}\right)(0.61 \mathrm{eV})$. However, it has a negative $\Delta_{S F}^{(1)}=-0.10 \mathrm{eV}$. We will not go over the molecules in details as for the 2B-doped analogues in SI. Instead, we focus more on the comparison of the two dopings.

Overall, the mirror relations between 2B- and 2N-dopings maintain. Figure 5(b) shows that the $K_{a, b} \mathrm{~s}, K_{H, L} \mathrm{~S}$, and $K_{H, L+1} \mathrm{~s}$ integrals of the $2 \mathrm{~N}$-doped phenanthrenes closely follow the same trends as the corresponding integrals of the $2 \mathrm{~B}$ analogues. Note that $K_{H, L+1}$ of $2 \mathrm{~N}$-doping corresponds to $K_{H-1, L}$ of $2 \mathrm{~B}$-doping. The AH pairing of occupied and unoccupied orbitals underlies the parallel changes between the two sets of integrals. The arguments on the $\mathrm{CT}$ energies of the $\mathrm{HOMO} \rightarrow \mathrm{LUMO}$ and $\mathrm{HOMO}-1 \rightarrow$ LUMO excitations of the $2 \mathrm{~B}$ doped phenanthrenes can be transplanted to the CT energies of the HOMO $\rightarrow$ LUMO and $\mathrm{HOMO} \rightarrow \mathrm{LUMO}+1$ excitations of the $2 \mathrm{~N}$-doped molecules. The characters of the $S_{1}$ states of the $2 \mathrm{~N}$-doped molecules are in good correspondence to those of the $2 \mathrm{~B}$ analogues, i.e., the $r_{H L} \mathrm{~S}$ and $f_{S_{1}} \mathrm{~s}$ of each pair of $2 \mathrm{~N}$ - and $2 \mathrm{~B}$-dopings are qualitatively similar. Again, the role of $\mathrm{HOMO}-1 \rightarrow$ LUMO in the $S_{1}$ states of the $2 \mathrm{~B}$ species is taken by $\mathrm{HOMO} \rightarrow \mathrm{LUMO}+1$. Natural orbitals (Figures S17, S21, S24, and S27) and bond lengths indicate that the 2Ndoped species share similar resonance structures in their ground states with the $2 \mathrm{~B}$ analogues (see Figure S18(b), (d), (f), (h), (j), and (l)). The good correlations between the molecular orbitals of the 2B- and 2N-doped species in Figures S16(o), S19(q), S20(o), S22(q), S25(o), and S28(s) underlie the mirror relations. Note that in Figure S28(s), the natural orbitals of PB2B7 are correlated with the canonical orbitals of PN2N7, as the HOMO-1 and HOMO of the former molecule are ambiguous. 


\section{Conclusions and further discussions}

So far, most of the theoretical studies on designing singlet fission chromophores are based on the two sites model. Despite its broad applicability, this model has its limit, that $S_{1}$ and $T_{1}$ shall arise from the same $\mathrm{HOMO} \rightarrow \mathrm{LUMO}$ excitation. This requirement cannot always be met, especially for molecules that possess close-lying HOMO-1 and HOMO, and/or close-lying LUMO and LUMO+1. The investigated 2B- and 2N-doped anthracenes and phenanthrenes feature such close-lying orbitals. Among the 24 molecules studied in this work, 8 cannot be described using the two sites model: AB1B8, AB4B8, AB4aB8 $a$, and PB3B6, and their 2N-doped analogues. Actually, taking the undoped anthracene as the reference, then only for those molecules with their $r_{H L}>20$, we may conclude that the model is applicable. For the others, the model becomes less and less valid as $r_{H L}$ decreases. As shown in the two tables of this work, there is a continuous spectrum of $r_{H L}$, and there is no clear-cut threshold to signify the validity of the two sites model. We have used $r_{H L}>1$ as a validity indicator. But this simply means that the molecules with $r_{H L}>1$ are better (but not necessarily adequately) described using the two sites model than those with $r_{H L}<1$.

Similar to the doping of acceptors to generate $p$-type semiconductors, the $2 \mathrm{~B}$-dopings systematically lower LUMO energies and reduce both HOMO-LUMO and HOMO-1-LUMO gaps. This reduced HOMO-1-LUMO gaps promote the HOMO-1 $\rightarrow$ LUMO configurations to compete with the $\mathrm{HOMO} \rightarrow \mathrm{LUMO}$ configurations to dominate the $S_{1}$ states. Triplet oneelectron excitation is of pure diradical character and the excitation energy is more dependent on the orbital energy gap involved in the excitation. Therefore, all $T_{1}$ states of the doped molecules have the largest contributions from $\mathrm{HOMO} \rightarrow$ LUMO excitations. Singlet oneelectron excitation involves an extra charge-transfer energy. Once the HOMO-1 $\rightarrow$ LUMO excitation involves a shorter distance and then lower energy charge transfer (CT) than the $\mathrm{HOMO} \rightarrow \mathrm{LUMO}$ excitation, and this difference in CT energies counterpoises the difference in orbital energy gaps, $S_{1}$ is dominated by HOMO-1 $\rightarrow$ LUMO. The two sites model then becomes inapplicable. Even if the HOMO-1 $\rightarrow$ LUMO configuration does not dominate $S_{1}$, 
it may have low enough energy to effectively interact with the HOMO $\rightarrow$ LUMO configuration and lower the $S_{1}$ energy. In the language of hole and particle orbitals, this mixing of configurations reflects the polarization of the hole orbital to minimize its exchange integral with the particle orbital. This polarization is realized through mixing HOMO-1 and HOMO, and it unfavorably reduces transition dipole between the hole and particle orbitals, and consequently the $S_{1}$ oscillator strength. The low $E\left(S_{1}\right)$ is disadvantageous for satisfying the primary thermodynamic criterion for singlet fission, Eq. 1. On the other hand, the low $S_{1}$ energy may be advantageous since the solar spectrum has higher irradiance at the low energy region of red visible and near infrared photons. The smaller HOMO-1-LUMO gap may also induce multiple diradical characters and a low-lying $T_{2}$ state, so that Eq. 2 is not satisfied. PB3B6 and PB2B7 are two typical examples.

Similar to the doping of donors to generate $n$-type semiconductors, the $2 \mathrm{~N}$-dopings systematically generate high-lying HOMOs, and reduce both HOMO-LUMO and HOMOLUMO+1 gaps. There is an interesting mirror relation in orbital energy changing: the decreases of LUMO energy induced by 2B-dopings are similar to the increases of HOMO energy induced by 2N-dopings. The reductions of HOMO-LUMO and HOMO-1-LUMO gaps of the 2B-doped molecules are hence similar to the reductions of HOMO-LUMO and HOMOLUMO+1 gaps of the corresponding $2 \mathrm{~N}$-doped molecules. Considering the alternant hydrocarbon pairing theorem, the CT energies of the $\mathrm{HOMO} \rightarrow \mathrm{LUMO}$ configurations of a pair of 2B- and 2N-doped molecules are comparable. The CT energies of the HOMO-1 $\rightarrow$ LUMO configuration of a $2 \mathrm{~B}$-doped molecule and the $\mathrm{HOMO} \rightarrow \mathrm{LUMO}+1$ configuration of the corresponding $2 \mathrm{~N}$-doped molecule are also comparable. Consequently, the conclusions about the 2B-doped molecules in the previous paragraph are generally transferrable to the $2 \mathrm{~N}$ doped molecules, with the role of $\mathrm{HOMO}-1 \rightarrow$ LUMO being taken by $\mathrm{HOMO} \rightarrow \mathrm{LUMO}+1$, and likewise, the polarization of hole orbital being replaced by the polarization of particle orbital. The polarization is realized through mixing LUMO and LUMO+1. There are hence additional mirror relations between 2B- and 2N-dopings in $S_{0}$ character (e.g., diradical char- 
acter), $S_{1}$ character (e.g., $r_{H L}$ and $\left.f_{S_{1}}\right), T_{2}$ character (e.g., low $E\left(T_{2}\right)$ induced by multiple diradical characters), and key exchange integrals $\left(K_{a, b}, K_{H, L}\right.$, and $K_{H-1, L}$-vs- $\left.K_{H, L+1}\right)$. Despite the general mirror relations, there must be exceptions. AN1 $a \mathbf{N 8} a$ is such one. The high electronegativity of the N-C-N fragment gives an ionic ground state, vs. the diradicaloid ground state of $\mathbf{A B 1} a \mathbf{B 8} a$. This difference makes the pair incomparable.

Before this study, we tended to think that in general, a large $K_{H, L}$ corresponds to a small $K_{a, b}$, and vice versa. This is because when HOMO and LUMO are distributed on the same atoms, their localized orbitals, through cancelation of amplitudes in $\frac{1}{\sqrt{2}}(\mathrm{HOMO} \pm \mathrm{LUMO})$, are distributed on different sets of atoms (e.g., starred and unstarred atoms of alternant hydrocarbons with antiferromagnetic coupling), and vice versa. This anticorrelation holds well in Figure 5(a) for the 2B- and 2N-doped anthracenes. The together increases of $K_{H, L}$ and $K_{a, b}$ from the undoped anthracene to $\mathbf{A B 1 B 8}$ and $\mathbf{A B N B N}$ result from the loosening of the $\mathrm{AH}$ orbital pairing in the doped molecules. The anticorrelation, however, does not hold in Figure 5(b) for the doped phenanthrenes. PB1 $a$ B8 $a$ is a typical example with both small $K_{H, L}$ and $K_{a, b}$. Actually, when HOMO and LUMO are evenly distributed in a large number of atoms, even if they have amplitudes on the same atoms, their exchange integral is attenuated to a small quantity. This situation of having small $K_{H, L}$ and $K_{a, b}$ shall be avoided in designing singlet fission chromophores. The relation between $K_{H, L}$ and $K_{a, b}$ is a good subject for a future study.

All the 2B-doped molecules have been previously studied in the context of designing singlet fission chromophores. Most of the $2 \mathrm{~N}$-doped molecules investigated here are not good chromophore candidates, except only AN1N8 and AN4N8. And the synthesizabilities of these molecules are unknown. More interesting results of this work, we believe, are the relations between 2B- and 2N-dopings, which can be generalized to di-acceptor and di-donor dopings, and the discussion of the applicability of the two sites model. Actually, the findings of this work are applicable for all systems with (pseudo)-degenerate HOMO and HOMO-1, and/or (pseudo-)degenerate LUMO and LUMO+1, regardless of the origin of the (pseudo- 


\section{Acknowledgements}

We thank York University for the start-up grant (TZ: 481333) and the Natural Sciences and Engineering Research Council (NSERC) of Canada (TZ: RGPIN-2016-06276) for financial support. We thank the Neese group at the University of Bonn for their continuous development of the ORCA program package. We thank the Gordon group at the Iowa State University for their continuous development of the GAMESS-US program package. This research was enabled in part by support provided by WestGrid (www.westgrid.ca), SciNet (www.scinethpc.ca), and Compute Canada (www.computecanada.ca). 


\section{Supporting Information}

See Supporting Information for comparison of calculations using cc-pVDZ and cc-pVTZ basis sets, calculated results of undoped anthracene and phenanthrene, detailed analyses of 2B-doped phenanthrenes, all plottings of orbitals, and coordinates of the investigated molecules. 


\section{References}

(1) Smith, M. B.; Michl, J. Singlet Fission. Chem. Rev. 2010, 110, 6891-6936.

(2) Smith, M. B.; Michl, J. Recent Advances in Singlet Fission. Annu. Rev. Phys. Chem. 2013, 64, 361-386.

(3) Michl, J. Unconventional Solar Energy: Singlet Fission. Mol. Front. J. 2019, 3, 84-91.

(4) Shockley, W.; Queisser, H. J. Detailed Balance Limit of Efficiency of P-N Junction Solar Cells. J. Appl. Phys. 1961, 32, 510-519.

(5) Hanna, M. C.; Nozik, A. J. Solar Conversion Efficiency of Photovoltaic and Photoelectrolysis Cells with Carrier Multiplication Absorbers. J. Appl. Phys. 2006, 100, 074510.

(6) Krishnapriya, K. C.; Musser, A. J.; Patil, S. Molecular Design Strategies for Efficient Intramolecular Singlet Exciton Fission. ACS Energy Lett. 2019, 4, 192-202.

(7) Japahuge, A.; Zeng, T. Theoretical Studies of Singlet Fission: Searching for Materials and Exploring Mechanisms. ChemPlusChem 2018, 83, 146-182.

(8) Casanova, D. Theoretical Modeling of Singlet Fission. Chem. Rev. 2018, 118, 71647207.

(9) Ito, S.; Nagami, T.; Nakano, M. Molecular Design for Efficient Singlet Fission. J. Photochem. Photobiol. 2018, 34, 85-120.

(10) Xia, J.; Sanders, S. N.; Cheng, W.; Low, J. Z.; Liu, J.; Campos, L. M.; Sun, T. Singlet Fission: Progress and Prospects in Solar Cells. Adv. Mater. 2017, 29, 1601652.

(11) Scholes, G. D. Correlated Pair States Formed by Singlet Fission and Exciton-Exciton Annihilation. J. Phys. Chem. A 2015, 119, 12699-12705. 
(12) Paci, I.; Johnson, J. C.; Chen, X. D.; Rana, G.; Popović, D.; David, D. E.; Nozik, A. J.; Ratner, M. A.; Michl, J. Singlet Fission for Dye-Sensitizerd Solar Cells: Can a Suitable Sensitizer be Found? J. Am. Chem. Soc. 2006, 128, 16546-16553.

(13) Minami, T.; Ito, S.; Nakano, M. Theoretical Study of Singlet Fission in Oligorylenes. J. Phys. Chem. Lett. 2012, 3, 2719-2723.

(14) Ito, S.; Minami, T.; Nakano, M. Diradical Character Based Design for Singlet Fission of Condensed-Ring Systems with 4n Electrons. J. Phys. Chem. C 2012, 116, 1972919736.

(15) Minami, T.; Nakano, M. Diradical Character View of Singlet Fission. J. Phys. Chem. Lett. 2012, 3, 145-150.

(16) Minami, T.; Ito, S.; Nakano, M. Fundamental of Diradical-Character-Based Molecular Design for Singlet Fission. J. Phys. Chem. Lett. 2013, 4, 2133-2137.

(17) Padula, D.; Omar, Ö. H.; Nematiaram, T.; Troisi, A. Singlet Fission Molecules among Known Compounds: Finding a Few Needles in a Hay Stack. Energy Environ. Sci. 2019, 12, 2412-2416.

(18) Schwerin, A. F.; Johnson, J. C.; Smith, M. B.; Sreearunothai, P.; Popović, D.; Černý, J.; Havlas, Z.; Paci, I.; Akdag, A.; MacLeod, M. K. et al. Toward Designed Singlet Fission: Electronic States and Photophysics of 1,3-Diphenylisobenzofuran. J. Phys. Chem. A 2010, 114, 1457-1473.

(19) Ryerson, J. L.; Schrauben, J. N.; Ferguson, A. J.; Sahoo, S. C.; Naumov, P.; Havlas, Z.; Michl, J.; Nozik, A. J.; Johnson, J. C. Two Thin Film Polymorphs of the Singlet Fission Compound 1,3-Diphenylisobenzofuran. J. Phys. Chem. C 2014, 118, 12121-12132.

(20) Johnson, J. C.; Nozik, A.; Michl, J. High Triplet Yield from Singlet Fission in a Thin Film of 1,3-Diphenylisobenzofuran. J. Am. Chem. Soc. 2010, 132, 16302-16303. 
(21) Johnson, J. C.; Michl, J. 1,3-Diphenylisobenzofuran: a Model Chromophore for Singlet Fission. Top. Curr. Chem. 2017, 375, 80.

(22) El Bakouri, O.; Smith, J. R.; Ottosson, H. Strategies for Design of Potential Singlet Fission Chromophores Utilizing a Combination of Ground-State and Excited-State Aromaticity Rules. J. Am. Chem. Soc. 2020, 142, 5602-5617.

(23) Baranac-Stojanović, M. Triplet-State Structures, Energies, and Antiaromaticity of BN Analogues of Benzene and Their Benzo-Fused Derivatives. J. Org. Chem. 2019, 84, $13582-13594$.

(24) Fallon, K. J.; Budden, P.; Salvadori, E.; Ganose, A. M.; Savory, C. N.; Eyre, L.; Dowland, S.; Ai, Q.; Goodlett, S.; Risko, C. et al. Exploiting Excited-State Aromaticity to Design Highly Stable Singlet Fission Materials. J. Am. Chem. Soc. 2019, 141, 1386713876 .

(25) Nagami, T.; Okada, K.; Miyamoto, H.; Yoshida, W.; Tonami, T.; Nakano, M. Molecular Design Principle for Efficient Singlet Fission Based on Diradical Character and Exchange Integral: Molecular Design Principle for Efficient Singlet Fission Based on Diradical Character and Exchange Integral: Multiple Heteroatom Substitution Effect on Anthracene. J. Phys. Chem. C 2020, 124, 11800-11809.

(26) Nagami, T.; Miyamoto, H.; Yoshida, W.; Okada, K.; Tonami, T.; Nakano, M. Theoretical Molecular Design of Phenanthrenes for Singlet Fission by Diazadibora-Substitution. J. Phys. Chem. A 2020, 124, 6778-6789.

(27) Malrieux, J. P.; Caballol, R.; Calzado, C. J.; de Graaf, C.; Guihéry, N. Magnetic Interactions in Molecules and Highly Correlated Materials: Physical Content, Analytical Derivation, and Rigorous Extraction of Magnetic Hamiltonians. Chem. Rev. 2014, 114, $429-492$. 
(28) Stuyver, T.; Chen, B.; Zeng, T.; Geerlings, P.; De Proft, F.; Hoffmann, R. Do Diradicals Behave like Radicals? Chem. Rev. 2019, 119, 11291-11351.

(29) Stoycheva, J.; Tadjer, A.; Garavelli, M.; Spassova, M.; Nenov, A.; Romanova, J. BoronDoped Polycyclic Aromatic Hydrocarbons: a Molecular Set Revealing the Interplay between Topology and Singlet Fission Propensity. J. Phys. Chem. Lett. 2020, 11, 13901396.

(30) Singh, S.; Jones, W. J.; Siebrand, W.; Stoicheff, B. P.; Schneider, W. G. Laser Generation of Excitons and Fluorescence in Anthracene Crystals. J. Chem. Phys. 1965, 42, $330-342$.

(31) Petrenko, T.; Krykova, O.; Neese, F.; Sokolowski, M. Optical Absorption and Emission Properties of Rubrene: Insight from a Combined Experimental and Theoretical Study. New. J. Phys. 2009, 11, 015001.

(32) McGlynn, S. P.; Azumi, T.; Kasha, M. External Heavy Atom Spin-Orbital Coupling Effect. V. Absorption Studies of Triplet States. J. Chem. Phys. 1964, 40, 507-515.

(33) Heinecke, E.; Hartmann, D.; Muller, R.; Hese, A. Laser Spectroscopy of Free Pentacene Molecules (I): the Rotational Structure of the Vibrationless $\mathrm{S}_{1} \rightarrow \mathrm{S}_{0}$ Transition. J. Chem. Phys. 1998, 109, 906-911.

(34) Zeng, T.; Hoffmann, R.; Ananth, N. The Low-Lying Electronic States of Pentacene and Their Roles in Singlet Fission. J. Am. Chem. Soc. 2014, 136, 5755-5764.

(35) Fuemmeler, E. G.; Sanders, S. N.; Pun, A. B.; Kumarasamy, E.; Zeng, T.; Miyata, K.; Steigerwald, M. L.; Zhu, X.-Y.; Sfeir, M. Y.; Campos, L. M. et al. A Direct Mechanism of Ultrafast Intramolecular Singlet Fission in Pentacene Dimers. ACS Cent. Sci. 2016, 2, 316-324. 
(36) Zeng, T.; Mellerup, S. K.; Yang, D.; Wang, X.; Wang, S.; Stamplecoskie, K. Identifying $(\mathrm{BN})_{2}$ pyrenes as a New Class of Singlet Fission Chromophores: Significance of Azaborine Substitution. J. Phys. Chem. Lett. 2018, 9, 2919-2927.

(37) Pradhan, E.; Bentley, J. N.; Caputo, C. B.; Zeng, T. Designs of Singlet Fission Chromophores with a Diazadiborinine Framework. ChemPhotoChem 2020, DOI:10.1002/cptc.202000081.

(38) Lee, S.; Filatov, M.; Lee, S.; Choi, C. H. Eliminating Spin-Contamination of Spin-Flip Time Dependent Density Functional Theory Within Linear Response Formalism by the Use of Zeroth-Order Mixed-Reference (MR) Reduced Density Matrix. J. Chem. Phys. 2018, 149, 104101.

(39) Lee, S.; Kim, E. E.; Nakata, H.; Lee, S.; Choi, C. H. Efficient Implementations of Analytic Energy Gradient for Mixed-Reference Spin-Flip Time-Dependent Density Functional Theory (MRSF-TDDFT). J. Chem. Phys. 2019, 150, 18411.

(40) Becke, A. D. Density-Functional Thermochemistry. III. The Role of Exact Exchange. J. Chem. Phys. 1993, 98, 5648-5652.

(41) Lee, C.; Yang, W.; Parr, R. G. Development of the Colle-Salvetti Correlation-Energy Formula into a Functional of the Electron Density. Phys. Rev. B 1988, 37, 785-789.

(42) Angeli, C.; Cimiraglia, R.; Evangelisti, S.; Leininger, T.; Malrieu, J.-P. Introduction of N-Electron Valence States for Multireference Perturbation Theory. J. Chem. Phys. 2001, 114, 10252-10264.

(43) Angeli, C.; Cimiraglia, R.; Malrieu, J.-P. N-Electron Valence State Perturbation Theory: a Fast Implementation of the Strongly Contracted Variant. Chem. Phys. Lett. 2001, 328, 297-305. 
(44) Angeli, C.; Cimiraglia, R.; Malrieu, J.-P. N-Electron Valence State Perturbation Theory: A Spinless Formulation and an Efficient Implementation of the Strongly Contracted and of the Partially Contracted Variants. J. Chem. Phys. 2002, 117, 91389153.

(45) Dunning, T. H. Gaussian Basis Sets for Use in Correlated Molecular Calculations. I. The Atoms Boron Through Neon and Hydrogen. J. Chem. Phys. 1989, 90, 1007-1023.

(46) Nakano, H.; Uchiyama, R.; Hirao, K. Quasi-Degenerate Perturbation Theory with General Multiconfiguration Self-Consistent Field Reference Functions. J. Comput. Chem. 2002, 23, 1166-1175.

(47) Miyajima, M.; Watanabe, Y.; Nakano, H. Relativistic Quasidegenerate Perturbation Theory with Four-Component General Multiconfiguration Reference Functions. J. Chem. Phys. 2006, 124, 044101.

(48) Ebisuzaki, R.; Watanabe, Y.; Nakano, H. Efficient Implementation of Relativistic and Non-Relativistic Quasidegenerate Perturbation Theory with General Multiconfigurational Reference Functions. Chem. Phys. Lett. 2007, 442, 164-169.

(49) Kawashima, Y.; Hashimoto, T.; Nakano, H.; Hirao, K. Theoretical Study of the Valence $\pi \rightarrow \pi^{*}$ Excited States of Polyacenes: Anthracene and Naphthacene. Theor. Chem. Acc. 1999, 102, 49-64.

(50) Schmidt, M. W.; Baldridge, K. K.; Boatz, J. A.; Elbert, S. T.; Gordon, M. S.; Jensen, J. H.; Koseki, S.; Matsunaga, N.; Nguyen, K. A.; Su, S. et al. General Atomic and Molecular Electronic Structure System. J. Comput. Chem. 1993, 14, 1347-1363.

(51) Gordon, M. S.; Schmidt, M. W. In Theory and Applications of Computational Chemistry: The First Forty Years; Dykstra, C. E., Frenking, G., Kim, K. S., Scuseria, G. E., Eds.; Elsevier: Amsterdam, 2005; Chapter 41, pp 1167-1189. 
(52) Neese, F. The ORCA Program System. WIRES Comput. Mol. Sci. 2012, 2, 73-78.

(53) Yamaguchi, K. In Self-Consistent Field: Theory and Applications; Carbó, R., Klobukowski, M., Eds.; Elsevier B. V.: The Netherlands, 1990; Chapter 20, pp 727-823.

(54) Flynn, C. R.; Michl, J. $\pi, \pi$-Biradicaloid Hydrocarbons. o-Xylylene. Photochemical Preparation from 1,4-Dihydrophthalazine in Rigid Glass, Electric Spectroscopy, and Calculations. J. Am. Chem. Soc. 1974, 96, 3280-3288.

(55) Bersuker, I. B. The Jahn-Teller Effect; Cambridge University Press: Cambridge, UK, 2006.

(56) Lee, J.; Jadhav, P.; Reusswig, P. D.; Yost, S. R.; Thompson, N. J.; Congreve, D. N.; Hontz, E.; Van Voorhis, T.; Baldo, M. A. Singlet Exciton Fission Photovoltaics. Acc. Chem. Res. 2013, 46, 1300-1311.

(57) Ovchinnikov, A. A. Multiplicity of the Ground State of Large Alternant Organic Molecules with Conjugated Bonds. Theor. Chem. Acc. 1978, 47, 297-304.

(58) Würfel, P. Physics of Solar Cells: From Basic Principles to Advanced Concepts; WILEY-VCH Verlag: Weinheim, 2009.

(59) Wen, J.; Havlas, Z.; Michl, J. Captodatively Stabilized Biradicaloids as Chromophores for Singlet Fission. J. Am. Chem. Soc. 2015, 13\%, 165-172.

(60) Akdag, A.; Havlas, Z.; Michl, J. Search for a Small Chromophore with Efficient Singlet Fission: Biradicaloid Heterocycles. J. Am. Chem. Soc. 2012, 134, 14624-14631.

(61) Zeng, T.; Ananth, N.; Hoffmann, R. Seeking Small Molecules for Singlet Fission: a Heteroatom Substitution Strategy. J. Am. Chem. Soc. 2014, 136, 12638-12647.

(62) Zeng, T.; Goel, P. Design of Small Intramolecular Singlet Fission Chromophores: An Azaborine Candidate and General Small Size Effects. J. Phys. Chem. Lett. 2016, 7, $1351-1358$. 
(63) Zeng, T. Through-Linker Intramolecular Singlet Fission: General Mechanism and Designing Small Chromophores. J. Phys. Chem. Lett. 2016, 7, 4405-4412.

(64) Japahuge, A.; Lee, S.; Choi, C. H.; Zeng, T. Design of Singlet Fission Chromophores with Cyclic (Alkyl)(Amino) Carbene Building Blocks. J. Chem. Phys. 2019, 150, 234306 .

(65) Ito, S.; Nakano, M. Theoretical Molecular Design of Heteroacenes for Singlet Fission: Tuning the Diradical Character by Modifying $\pi$-Conjugation Length and Aromaticity. J. Phys. Chem. C 2015, 119, 148-157. 


\section{TOC Graphic}

\section{TOC Graphic}

\section{For Table of Contents use only}

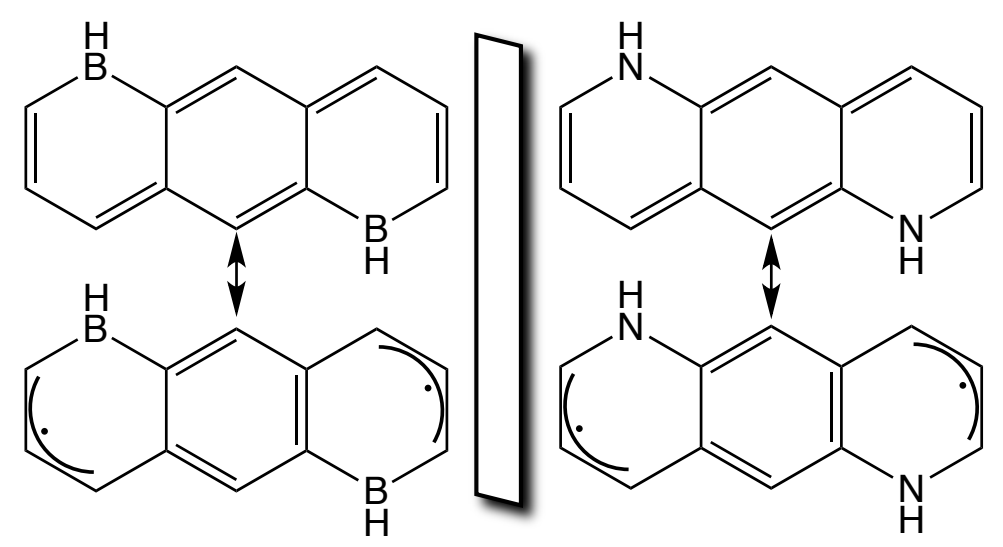

Diboron- and diaza-dopings in anthracene and phenanthrene exhibit a series of mirror relations. 